Portland State University

PDXScholar

$5-22-1986$

\title{
Adsorption of Aminopyridines to Phosphatidylserine Vesicles
}

Colin Wright

Portland State University

Follow this and additional works at: https://pdxscholar.library.pdx.edu/open_access_etds

Part of the Biology Commons

Let us know how access to this document benefits you.

\section{Recommended Citation}

Wright, Colin, "Adsorption of Aminopyridines to Phosphatidylserine Vesicles" (1986). Dissertations and Theses. Paper 3686.

https://doi.org/10.15760/etd.5570

This Thesis is brought to you for free and open access. It has been accepted for inclusion in Dissertations and Theses by an authorized administrator of PDXScholar. Please contact us if we can make this document more accessible: pdxscholar@pdx.edu. 
AN ABSIRACI OF THE IHESIS OF Colin wright for the Master of Science in Physics presented May 22, 1986.

Iitle: Adsorption of Aminopyridines to Phosphatidylserine Uesicles.

APFRDUED BY MEMBERS OF THE THESIS COMMITIEE:

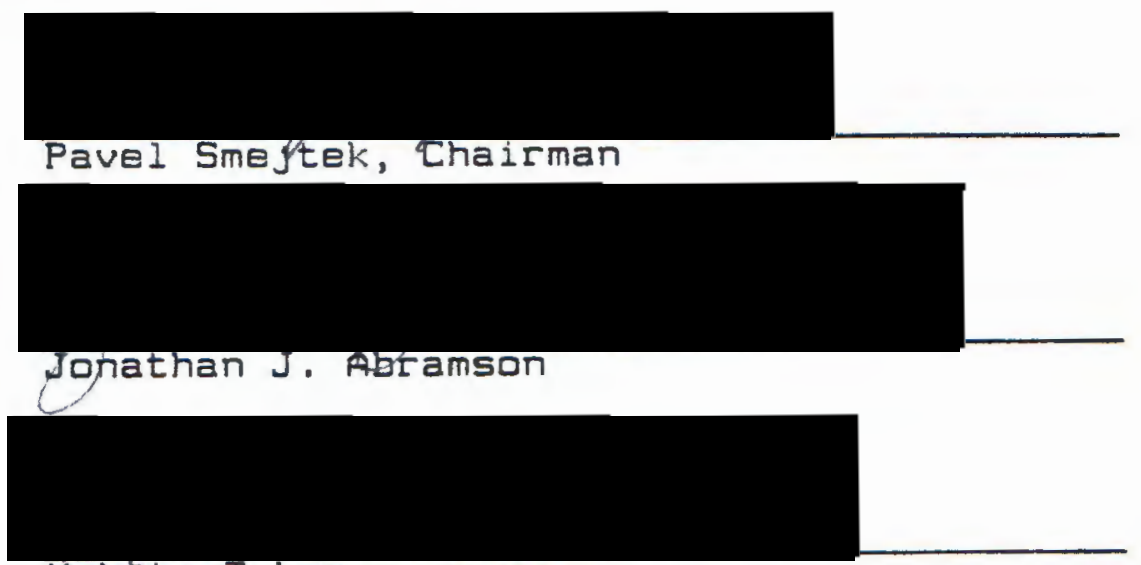

Makoto-Iakeo

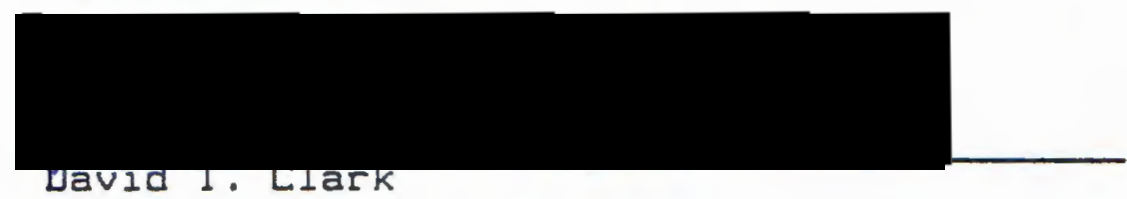

Intrinsic association constants were determined for a series of aminopyridines on phosphatidylserine vesicles, through determination of electrophoretic mobility. The magnitudes of the constants were such that depolarization of the nerve terminal through binding to negative phospholipids seems unlikely to occur. The aminopyridines all had association constants between one half and one 
sixtieth the association constant of calcium. The aminopyridines probably block potassium channels in their enhancement of synaptic transmission.

An interesting correlation was noted in that the ranking of the aminopyridines in terms of their affinity For these lipids was approximately the same as the ranking of their enhancement of synaptic transmission in a wide variety of biological preparations. A possible mechanism for this correlation is that adsorption of these drugs to lipid membranes is the initial and rate-limiting step in their action. 
ADSORPIION OF AMINOPYRIDINES TO

PHOSPHATIDYLSERINE UESICLES

by

COLIN WRIGHT

A thesis submitted in partial fulfillment of the requirements for the degree of

\author{
MASTER OF SCIENCE \\ in \\ PHYSICS
}

Portland State University

1986 
TD THE GFFICE DF GRADUATE STUDIES AND RESEARCH:

The members of the Committee approve the thesis of

Colin Wright presented May ट2, 1986.
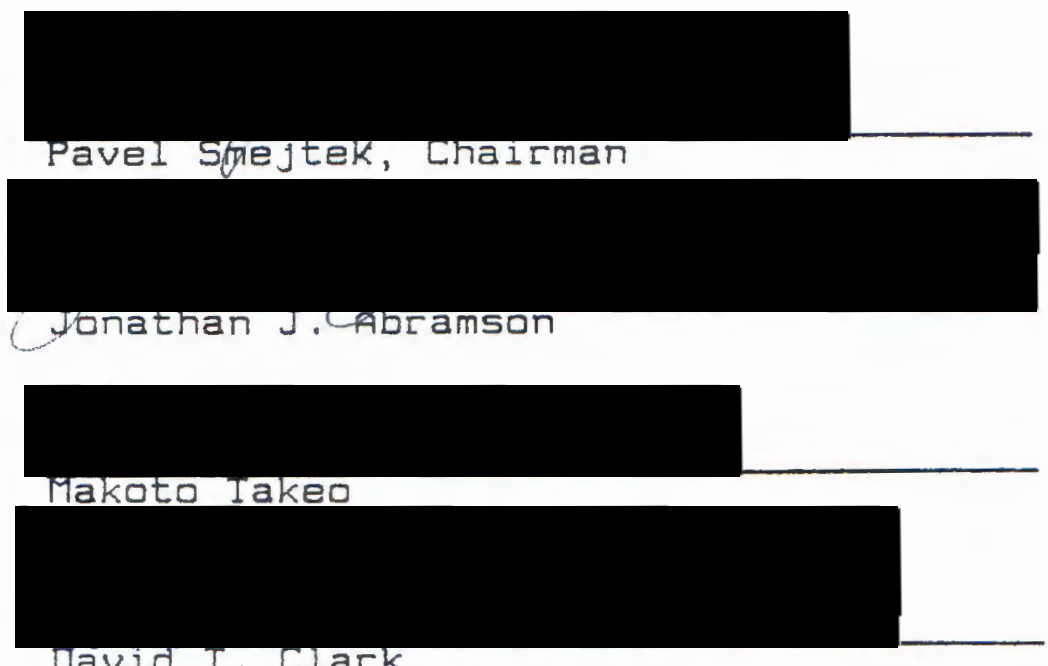

APPROUED :

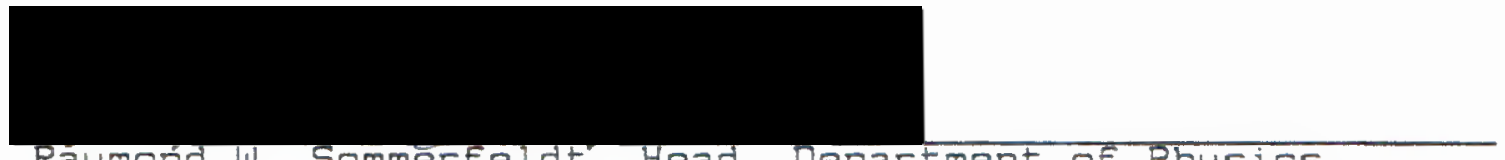

Raymond w. Sommerfelde, Head, Department of physics

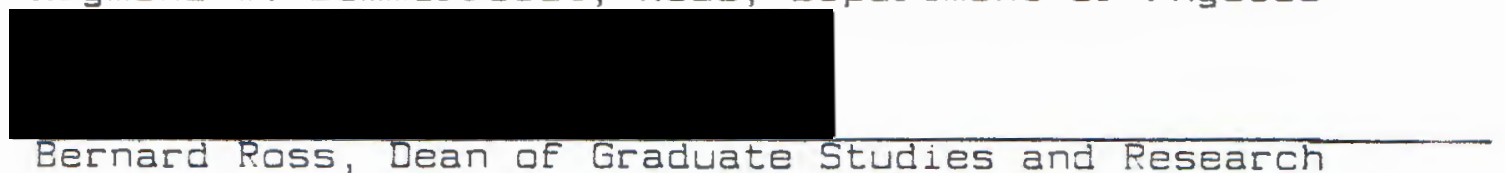




\section{ACKNOWLEDGEMENTS}

First and Eoremost, I wauld like to thank Dr. Smejtek Eor nourishing my growth as a person and as a scientist during my time at Portland State. I appreciate his gaodhumoredness and the Ereedom he allowed me to explore related topics. Regarding the thesis proper, Alevardios Oxyzoglou's data on calcium and 3,4-diaminopycidine is ackinowledged, while Bill Barstad's willingness to help with the computer and lab equipment is not forgotten.

In thanking my cammittee, I would like in particular to extend my gratitude to DI. Takeo for his emphasis =n clarity and quality. Good soientific writing is diEEz=ult to achieve and I have learned from his prodings ir that direction. The cloudiness that remains is of course entirely my own doing.

Among the graduate students, Jcel Nissen, Ice Waizers and Hirashi Takeo provided especialiy warm Eliendships. Megan Lerchen conteibuted much support during the iang hours of data coilecting, while my mother, though many miles away, remained a constant scurce of encouragement. Finaliy, I would like to thani: Dr. SommezEeldt and the entire Physics department Ear providing sufport and a reianing atmosphere in which to warl: and learn. 
IABLE OF CONTENTS

PAGE

ALKNOWLEDGEMENTS $\ldots \ldots \ldots \ldots \ldots \ldots \ldots \ldots \ldots \ldots$

LIST OF TABLES

LIST OF FIGURES $\ldots \ldots \ldots \ldots \ldots \ldots \ldots \ldots \ldots \ldots \ldots \ldots \ldots \ldots \ldots$

CHAPTER

I INTRODUCTION $\ldots \ldots \ldots \ldots \ldots \ldots \ldots \ldots \ldots \ldots$

Aminopyridines $\ldots \ldots \ldots \ldots \ldots \ldots \ldots$ 4......

I I COMPUTATIONAL AND EXPERIMENTAL METHODS ... 10

Definition of binding constant ....... 10

The Helmholtz-Smoluchowski equation ... 13

Description of adsorption model ...... 18

Computational methods $\ldots \ldots \ldots \ldots \ldots \ldots$ 19

Experimental methods $\ldots \ldots \ldots \ldots \ldots \ldots \ldots \quad 21$

I I RESULTS AND DISCUSSION $\ldots \ldots \ldots \ldots \ldots \ldots \ldots$ 26

Summary of results $\ldots \ldots \ldots \ldots \ldots \ldots \ldots$ 26

The surface potential theory $\ldots \ldots \ldots \ldots$. 28

A model of the calcium channel ....... 32

IU CAN AMINOPYRIDINES DEPOLARIZE PRESYNAPTIC TERMINALS? ................ 39

Conclusion ................ 41

REFERENCES $\ldots \ldots \ldots \ldots \ldots \ldots \ldots \ldots \ldots \ldots \ldots \ldots \ldots \ldots \ldots \ldots$

APPENDIX A $\ldots \ldots \ldots \ldots \ldots \ldots \ldots \ldots \ldots \ldots \ldots \ldots \ldots \ldots$

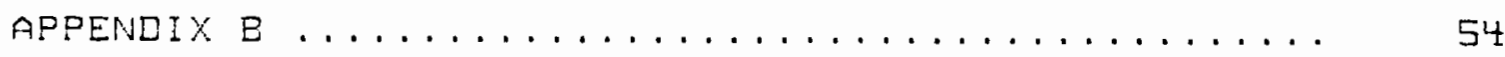




\section{LIST OF TABLES}

TABLE

PAGE

I Experimental association constants ........

I Theoretical vs experimental zeta potentials

for calcium ...................

55

II Theoretical vs experimental zeta potentials

for 3,4-diaminopyridine .............

IU Theoretical vs experimental zeta potentials

For 4,5 -diaminopyrimidine (pH 5.5) ......

$\cup$ Theoretical vs experimental zeta potentials

for 4,5-diaminopyrimidine $(p H$ 6.6) ......

U Theoretical vs experimental zeta potentials

For 4-aminopyridine ..............

UI Theoretical vs experimental zeta potentials

for 3-aminopyridine $(p H 5.4) \ldots \ldots \ldots \ldots$

UII Theoretical vs experimental zeta potentials

For 3-aminopyridine cpH 6.6$) \ldots \ldots \ldots \ldots$

IX Theoretical vs experimental zeta potentials

for Z-aminopyridine $\mathrm{c} p \mathrm{H} \quad 6.2 ; \ldots \ldots \ldots \ldots$

$x$ Theoretical vs experimental zeta potentials

Eor 2-aminopyridine $6 \mathrm{pH} 7.4 \mathrm{f} \ldots \ldots \ldots \ldots$

XI Theoretical vs experimental zeta potentials

For 4-dimethylaminopyridine ..........

XII Theoretical vs experimental zeta potentials 
For 4-aminopyridine methiodide ......... 


\section{LIST DF FIGURES}

F IGURE

PAGE

1. A representative synapse $\ldots \ldots \ldots \ldots \ldots \ldots \ldots \ldots \ldots \ldots$

ᄅ. Preservation of synaptic transmission by

3,4-diaminopycidine in low calcium solution

3. Aminopyridines studied and their

abbreviations $\ldots \ldots \ldots \ldots \ldots \ldots \ldots \ldots$

4. Phosphatidylserine and other molecules

mentioned in the text $\ldots \ldots \ldots \ldots \ldots \ldots$

5. Postulated potential profile across an axon

membrane $\ldots \ldots \ldots \ldots \ldots \ldots \ldots \ldots \ldots \ldots \ldots \ldots \ldots \ldots \ldots \ldots$

6. Reconstruction of events at synaptic

transmission $\ldots \ldots \ldots \ldots \ldots \ldots \ldots$

7. Hypothetical model of calcium channel .......

B. Adsorption isotherm for

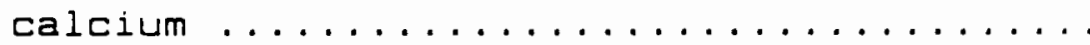

9. Adsorption isotherm for

3,4-diaminopyridine $\ldots \ldots \ldots \ldots \ldots \ldots$

10. Adsorption isotherm for

4,5-diaminopyrimidine $(p H 5.5) \ldots \ldots \ldots$

11. Adsorption isotherm For

4,5-diaminopyrimidine (pH 6.6$) \ldots \ldots 60$

12. Adsorption isotherm for

4-aminopyridine ................. 
13. Adsorption isotherm for

$$
\text { 3-aminapyridine }(p H 5.4) \ldots \ldots \ldots . \ldots \ldots
$$

14. Adsarption isotherm far

$$
\text { 3-aminopyridine }(p H 6.6) \ldots \ldots \ldots \text { 6E }
$$

15. Adsorptian isotherm for

$$
\text { 2-aminopyridine }(p H 6.2) \ldots \ldots \ldots
$$

16. Adsorption isotherm for

$$
\text { 2-aminopyridine }\{p H 7.4\} \ldots \ldots 70
$$

17. Adsorption isotherm far

4-dimethylaminopyridine .............

18. Adsorption isotherm for

4-aminopyridine methiode ............ 74 


\section{CHAPTER I}

\section{INTRODUCTION}

Ihis thesis describes a study of the adsorption of a series of aminopyridines to phosphatidylserine multilamellar vesicles. Phosphatidylserine is one of the major negatively charged lipids that have been found in cell membranes. Multilamellar vesicles are the thermodynamically stable form of a suspension of these lipids in water. The rationale behind the study is to gain insight into the biological mode of action of the aminopyridines. They have been found to facilitate synaptic transmission, that is the jumping of a neural impulse from one neuron to the next.

The underlying problem is essentially this: how does the incoming action potential liberate the neurotransmitter, the chemical substance that carries the signal across the synaptic cleft? (The synaptic cleft is the small region (40-100 angstrams) between the end of one nerve cell and the start of another. See figure 1.3 


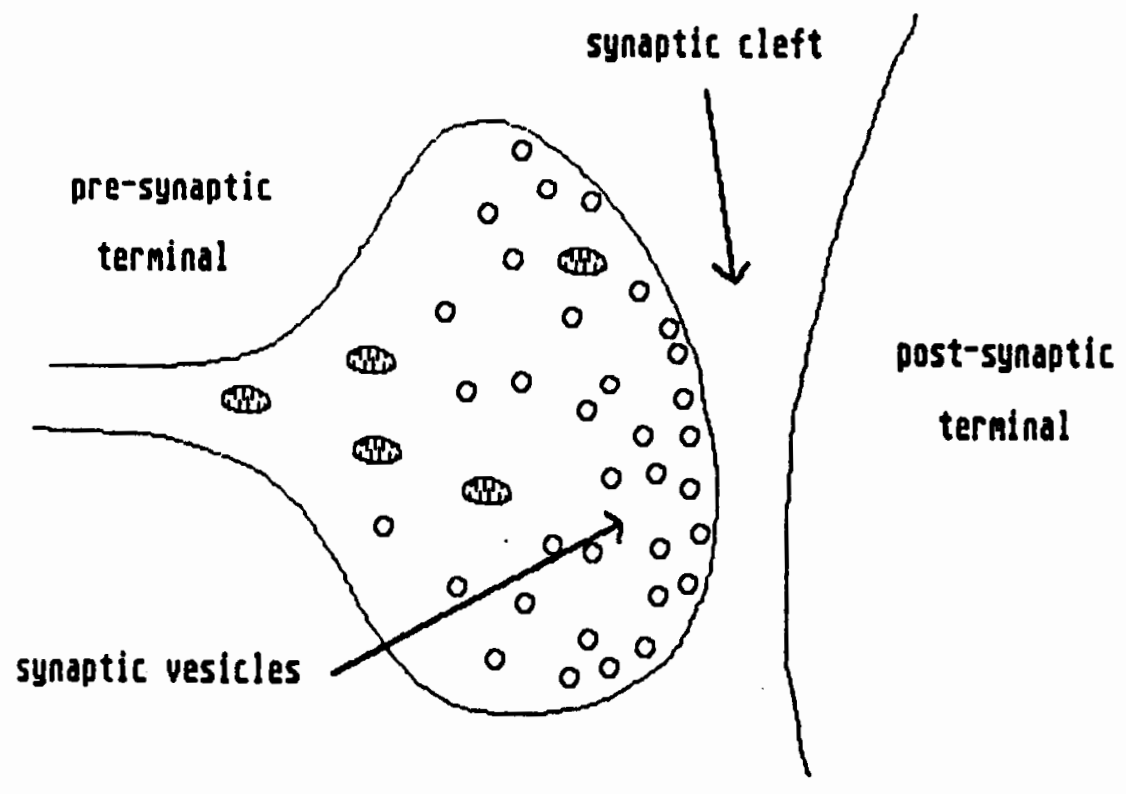

Figure 1. A representative synapse

What is known about this transmission process is that inside the presynaptic nerve terminal are minute synaptic vesicles, each about $250 \mathrm{~A}$ in radius, and Eilled with several thousand neurotransmitter molecules (1). The inside of the neuron is held at about -60 to $-70 \mathrm{millivolts}$ with respect to the outside of the neuron. When the incoming signal, the propagating action potential, "depolarizes" (i.e. makes more positive) the inside of the nerve terminal, the vesicles adjacent to the cell membrane Euse with it, liberating the transmitter into the synaptic cleft. From here, the transmitter molecules diffuse to the postsynaptic membrane where they activate a response.

Katz and Miledi suggested correctly that intracellular calcium is the determinant for transmitter release (2). When the action potential arrives, the permeability of the 
membrane to calcium increases rapidly, calcium flows into the neuron and the vesicles are released. If no calcium is available in the external solution, no vesicles are released when the membrane is depolarized.

Various aminopyridines have been found to enhance this synaptic transmission process (e.g. see reference 3), i.e. higher postsynaptic potentials are generated. They have also been found to be able to substitute for a certain proportion of the extra-cellular calcium. That is, a lower concentration of external calcium can be compensated for by a small amount of aminopyridine, to give the same postsynaptic response. Figure 2 presents these ideas in the form of experimental data collected by Matsomato and Riker Erom the Oregon Health Sciences University. The traces represent the average postsynaptic response as the external calcium concentration is reduced as indicated. In the top set, we see almost complete transmission Eailure at about $0.5 \mathrm{millimolar}$ calcium, whereas, in the bottom set, with the addition of 0.1 millimolar of an aminopyridine cin this case, 3,4-diaminopyridinej transmission Failure occurs at the much lower level of $0.1 \mathrm{mM}$ calcium.

This might suggest a competition between the aminopyridine and the calcium for a common site or domain on the presynaptic external membrane terminal (4). On the other hand, some studies suggest that the aminopyridines act intracellularly 65), where the calcium concentration is 

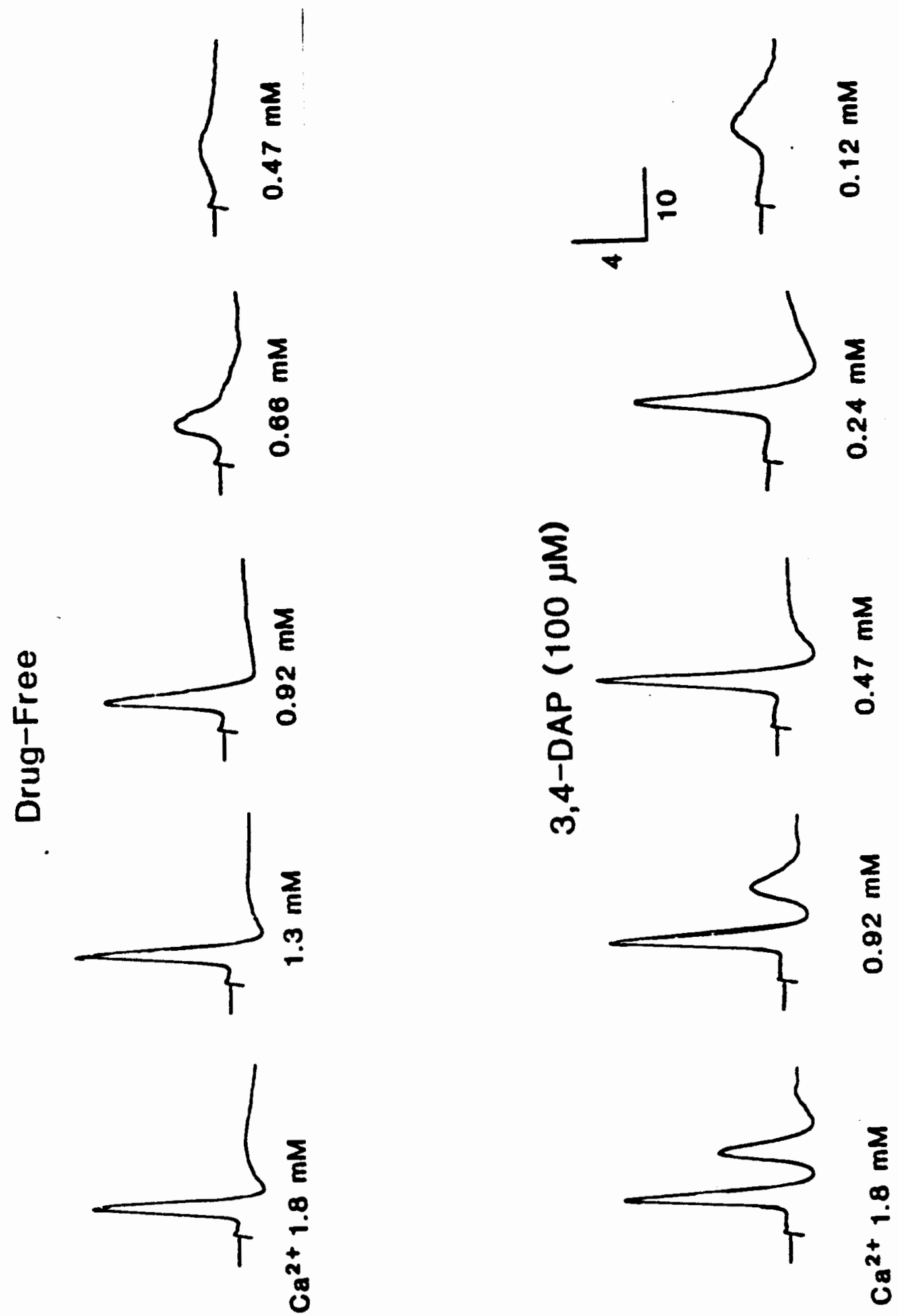

Figure 2. Preservation of synaptic transmission by 3,4-diaminopyridine in low calcium solution. Traces are the extracellular recordings of postganglionic responses to preganglionic stimulation in the same isolated bullfrog sympathetic ganglion. From Matsumoto and Riker (3). 
kept at very low concentrations by active mechanisms of the cel1. By interfering with potassium channels, the aminopyridines may be altering the action potential with the result that more calcium enters the cell.

To test the former possibility, that of a competition For sites on the presynaptic membrane, a study of the binding of calcium and aminopyridines to a representative lipid was undertaken. Phenomenological association constants were determined for each of 7 aminopyridines plus 1 analog. CSee Figures 3 and 4 for molecular structures and abbreviations.used.J The magnitudes of these constants were fairly low, but their order correlated remarkably well with the biological potency of this series of aminopyridines. A proposed mechanism is given to explain this correlation in the conclusion of the thesis. The main result too is annunciated there, that it does not appear likely that aminopyridines can bind sufEiciently strongly to lipids to account for their remarkable properties.

This chapter concludes with a brief and slightly technical resume of the history of aminopyridines in medical science in an attempt to put in perspective the problem we are dealing with. Chapter two describes both the computational and the experimental aspects of the determination of the association constants. With regard to the former, a short, non-rigorous introduction to 
electrical double layer theory is given in Appendix $A$.

This forms an essential part in accounting for the adsorption of a charged species from solution anto a surface fin our case, lipid vesicles). Chapter three begins with a summary of the experimental results (the individual data are relegated to Appendix B), and is followed by a discussion. Finally, a conclusion is offered in chapter Eive.

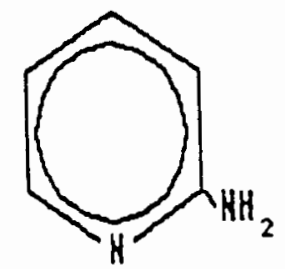

2-aninopyridine (2-AP)

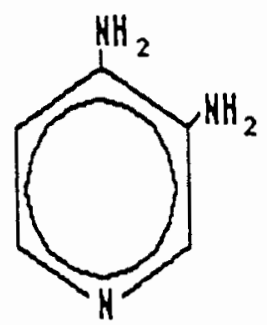

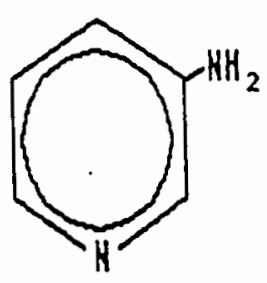

3-aminopyridine (3-AP)

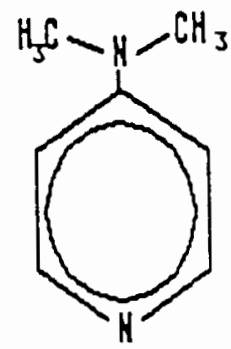

3,4-diaminopyridlne 4-dimethylaminopyridine $(3,4-D A P)$ (4-DMAP)

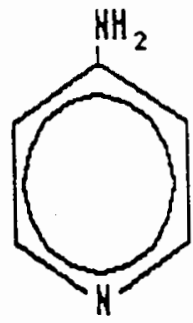

4-aminopyridine $(4-A P)$

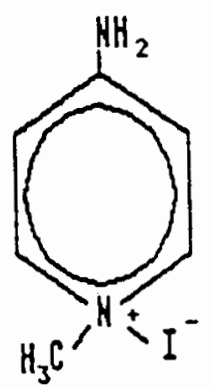

4-aminopyridine methiodide (4-APMI)

Figure 3. Aminopyridines studied and their abbreviations 


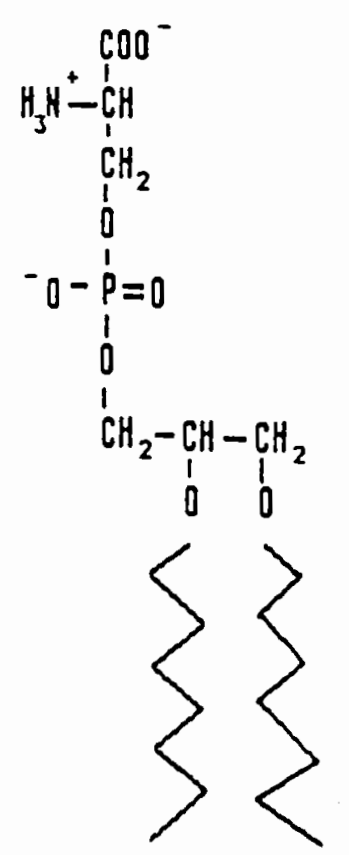

phosphatidylserine (PS)<smiles>c1cncnc1</smiles>

\section{4,5-diaminopyrimidine $(4,5-D R P M)$}

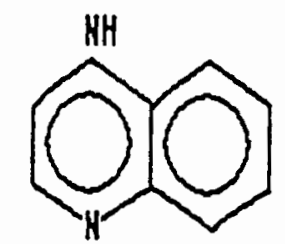

4 -aminoquinoline $(4-g Q)$<smiles>N=C(N)N</smiles>

guanidine

Figure 4. Phosphatidylserine and other molecules mentioned in the text.

\section{AMINOPYRIDINES}

Many of the pharmacological properties of aminopyridines, including their vasopressor and convulsant actions, were described by Dohrn in 1924 (6). However, over 30 years elapsed until there were further studies on the action of aminopyridine on nerve-muscle preparations. These studies clarified that aminopyridines facilitate transmitter release, not only at the neuromuscular junction but also at a wide range of synapses. Work, particularly in France, culminated in the discovery that aminopyridines were selective blockers of potassium channels (Pelhate et 
al (1974) (7)). This provided an explanation for some of the pharmacological actions of the drugs and, furthermore provided researchers with a useful probe of excitable membranes. In this latter respect, aminopyridines offered a number of advantages over tetraethylammonium (TEA), the classical inhibitor of potassium conductance. Today, interest in these compounds has increased enough to warrant an international conference devoted to their actions. (The conference, which was held in 1981 had over 100 participants from 25 different countries (8)).

Extensive reviews exist that summarize the various studies that have been performed on many different biological preparations with aminopyridines $(9,10)(\mathrm{e} . \mathrm{g}$. see Glover ( 9 ) with over 200 references). In particular, it is now clearly estabished that aminopyridines block potassium channels in a wide range of excitable membranes (Erog sciatic nerve, cat hind paw, cockroach axon, squid giant axon, etc), irrespective of the type of transmitter substance (e.g. acetylcholine, noradrenaline, etc.). They are generally mare potent than IEA and have different blocking kinetics $(11,12)$. The aminopyridines also have valuable potential clinical applications. They have been used as an antagonist to muscle paralysis in botulism and various muscular diseases, such as Eaton-Lambert syndrome (13).

Questions remain concerning specific effects of the 
aminopyridines. For instance, in squid, 4 -AP causes depolarization and induces repetitive Firing (14). In some experiments, in high concentrations, they were found to increase spontaneous (that is, in the absence of direct stimulation) transmitter release (15), and, in others, to produce repetitive post-synaptic potentials, in response to single shock stimulation (in frog (16)). Thus, in some preparations, they increase membrane excitability. In addition, in frog sartorius, low doses of 4 -AP enhance twitching without modifying the action potential (17), while in mouse diaphragm 4 -AP prolongs action potential without modifying muscle contractility (18). Finally, Riker and Matsumoto noticed no broadening of action potentials in the individual nerve cells that contributed to the averaged traces of Figure 2 ( 3 ).

These kinds of studies have led certain researchers to propose new modes of action for the aminopyridines. For our purposes we can quote Kim et al. (19), who suggested that there may be two possible ways in which quaternary ammonium compounds could promote entry of calcium into the nerve terminal: "(1) through increased duration of the action potential, and ( 2 ) by reduction in the electrostatic screening of fixed negative charges an the nerve membrane." This thesis could be considered as arising to test this latter possibility. 


\section{CHAPTER I I}

In this chapter, we begin by defining the binding constants that we are setting out to measure. The means of measurement will be through determination of electrophoretic mobility. Consequently, we give a description of the Helmholtz-Smoluchowski equation which is necessary to relate the mobility to an electrical parameter, the electrokinetic or zeta potential. From Apendix $A$, which contains a short introduction to the GouyChapman theory of the electrical double layer, we are then able to calculate surface charge densities from the zeta potentials. Finally, a Langmuir adsorption isotherm is described from which we can obtain the binding constants Erom the surface charges. Details of the computation are fallowed by a section on the experimental methads.

\section{DEFINITION OF BINDING CONSTANT}

To quantify the postulated binding, we use the equilibrium binding constants defined by the one-to-one reaction, 


$$
A P(a q)+P S(m e m b) \Rightarrow A P-P S(m e m b)
$$

representing the adsorption of the aminopyridine onto the phosphatidylserine membrane. Here AP-PS represents the bound aminopyridine phosphatidylserine complex. Ihus, we have, for the equilibrium binding constant, $K$,

$$
X=\frac{(A P-P S)}{[A P]_{0}(P S)}
$$

where the parentheses represent surface concentrations on the membrane and the square brackets aqueous concentrations. A source of ambiguity arises here, in that part of the binding is electrostatic in origin--the membrane will be negatively-charged, the aminopyridines univalent cations. Because the electrostatic contribution will depend on the state of charge of the membrane, we define $k$ to be the "intrinsic" binding constant by correcting for the electrostatic attraction with a Boltzmann Eactor applied to the aminopyridine bulk concentration. That is,

$$
[A P]_{0}=[A P]_{B} \exp \left(-e \cup \sigma / k_{B} T\right)
$$

where $[A P]_{0}$ is the concentration adjacent to the surface (where the potential is $V_{0}$ and will be negativej, [AP] $]_{B}$ is the concentration in the bulk solution (where the potential will be zeral, and $e, k_{B}$ and $I$ are the proton charge, Boltzmann constant and absoiute temperature respectively. 
Io measure $K$ we will need some means of measuring the surface concentration of adsorbed aminopyridine on phosphatidylserine membranes. Io this end, we are aided by the fact that phosphatidylserine forms small spherical vesicles--ready-made membrane surfaces, composed of bimolecular layers--when shaken in an aqueous medium. The vesicles are visible under a microscope, and since they are negatively charged, they will drift in an applied electric field. Furthermore, since the aminopyridines are univalent cations, on adsorption they will tend to neutralize the lipid vesicles. The stronger the adsorption process cthe higher the value of $\mathrm{K}$ ), the more aminopyridine and positive charge on the vesicle surface at any one time and hence the slower the vesicle will move. Thus we have a way of comparing the actions of a variety of aminopyridines. A stopwatch and a graduated eyepiece will provide us with the raw data, i.e. the drift velocities, necessary to quantify the amount of binding. With an appropriate model we are then able to calculate the equilibrium binding constants we seek.

Already, in our above discussion of the Boltzmann factor, we have come into contact with the Gouy-Chapman theory of the electrical double layer--the attraction of mobile ions to a charged surface. This might be a good time to refer the interested reader to Appendix $A$, where a brief description of the theory and the mathematical 
equations that we will be using later are developed. Important for the next section is the notion of the "thickness" of the diffuse layer, the Debye screening length.

\section{THE HELMHOLIZ-SMOLUCHDWSK I EQUATION}

In general, there is a very complicated relationship between the charge on a particle and the drift velocity it will attain in an applied electric field. If we were dealing with, say, a charged bubble falling through an electric field, then our analysis wauld be simple. Here the Stokes' resisitance (For low Reynolds number) balances the electrical force,

$$
E \pi \eta a v=D E
$$

or,

$$
\mu=v / E=Q /(5 \pi \eta a)
$$

where $\mu$ defines the mobility, $\eta$ is the viscosity of the medium and a the particle radius.

However, in our case, the above relationship is invalid because we would be neglecting the effects of the diffuse cloud of oppositely-charged ians that will tend to collect around the particle. (Even in pure, distilled water there will be ions due to the dissociation of the water molecules.) The cloud will tend to move in the opposite direction to the particle in an applied electric 
Field, and thus the particle will effectively have to move "upstream". This is called the electrophoretic retardation effect. Furthermore, the "center of gravity" of the charged cloud will tend to lag behind the "center of gravity" of the particle, thus creating an additional

force. This is sometimes referred to as the retardation effect. Because of these complications, exact solutions to the hydrodynamic equations of motion are few, and then only under restrictive assumptions. The reader is referred to Qverbeek and Wiersema's article for more detailed discussion (20).

Fortunately, in the case when the "thickness" CDebye length) of the diffuse cloud is small in comparison to the radius of the particle, simplifications occur. First of all, we can approximate the natural spherical geometry of the particle localiy as a flat surface and proceed to make use of the results of Gouy-Chapman theory. Secondly, we can neglect the retardation effect in the calculation of the particle's motion.

Furthermore, we can arrange to reduce the "thickness" of the diffuse double layer, simply by adding more electrolyte to the solution. In our case, the vesicles are approvimately $1-10$ micrometers in diameter and, with $0.1 \mathrm{M}$ background electrolyte, the double layer "thichness" will be less than 10 angstrams. Thus, we have a ratio of particle size to double-layer "thichness" of 
more than one thousand to one, and the flat geometry will introduce only very small errors.

Armed then with our thin double layer, we can proceed to obtain a simple relationship between the particle mobility and its charge, first obtained by Helmholtz in 1879 and Smoluchowski in 1903. Actually the electric potential at the particle surface will turn out to be a more convenient variable than the particle charge, although here we must be careful by what we mean by "particle surface." It is a long accepted part of classical hydrodynamics that the fluid velocity at the surface of a rigid particle in a viscous medium is zero. Here we mean both the normal component of velocity and also the tangential component (the latter stipulation being the socalled non-slip hypothesis.) Thus, at least the layer of water immediately adjacent to the particle will stick to it, and the radius of our particle will have increased. The electric potential at this "surface of shear", is the definition of the electrokinetic or zeta potential $(\zeta)$. The zeta potential is what is measured in these experiments, not the surface potential from which it can have differ substantially. Assumptions must be made to relate these two potentials. In concordance with other workers in the field (21), we take the distance to the plane of shear to be 2 angstroms. We note, however, that this distance is a function of ionic strength for possibly, 
electric field strength (22)y, and that Davies and Rideal treat the case of a diffuse rather than a sharp plane of shear (23). )

continuing with the derivation, then, with the flat geometry, and $x$ representing the distance normal to the charged surface, the external force exerted on a layer of liquid of thickness dx is $\rho E d x$, with $\rho$ the space charge density (which moves with the particle: and $E$ the electric Field strength. Ihis farce will be purely balanced by the viscous force on the same shell (no pressure gradient need be invoked to satisfy the equations of motiony, so that

$$
\eta d^{2} v / d x^{2}=\rho E
$$

with $\eta$ the bulk viscosity and $v$ the Eluid velucity. However, since the space charge density is given by the Foisson equation, we have (in MkS units)

$$
\left.\eta d^{2} v / d x^{2}=c-\epsilon d^{2} \psi_{i d x^{x}}\right) E
$$

$c \in=\epsilon_{0} \epsilon_{r}$ is the product of the permittivity of Eree space and the dielectric constant and $\psi$ is the electic potential.j Two integrations and the use of the boundary conditions $\psi, d \psi / d x, v$, and $\dot{d}, \mathrm{~d} d x$ all equal to zero at iarge distances from the particle leave ws with

$$
v=-\imath \epsilon \psi, \eta, E
$$

The action of the applied field will be to impart a 
velocity, $U$, to the particle. On a coardinate system fixed to the particle, the fluid velocity at large distances will be $-U$ and we then have

$$
\mu=U / E=\epsilon \zeta / \eta
$$

the Helmholtz-Smaluchowski equation relating the mobility of our charged particle, with $\zeta$, the zeta potential, a result independent of the particle size.

For completeness, we mention that in the case when the size of the particle is much smaller than the diffuse double layer thickness, the value for the mobility is two thirds as much and can be shown (24) to reduce to $0 / 6 \pi \eta$ a, the Stokes' result mentioned at the start of this section. In the intermediate case, when neither particle size nor diffuse layer thickness dominate, there is no closed form solution, but the theory has been given by Henry and is included in Rice and Nagasawa's book (25), alang with a good discussion of inertial and relaxation effects.

So Ear then, we have an expression far the potentia? at the plane of shear. Our next step will be to relate the zeta potential (via the surface potential) to the surface charge on the particle and thence to the amount of bound aminopyridine. With an appropriate adsorption model, we can then extract the binding constants we are looking for. 
DESCRIPTION OF ADSORPTION MODEL

In surface science the relation between the amount of adsorbed material and the amount in solution is called the adsorption isotherm. One of the simplest of these is the Langmuir adsorption isotherm, developed in 1917, which provides a fixed lattice onto which the adsorbate binds. By simple kinetic arguments, the fraction of bound sites, $\theta$, will be proportional to the free ionic concentration at the membrane surface, in our case [AP]⿻, $(i . e$. the bulk concentration times a Boltzmann factor). $\theta$ will also be proportional to the fraction of free sites available to bind, $(1-\theta)$. The intrinsic binding constant, $k$, is then the proportionality constant,

$$
\theta=k[A]_{0}(1-\theta)
$$

This can be seen to be equivalent to our previous definition of $k$, equation ( 1 ) above, by dividing this latter equation through by the total number of binding sites.

It is easy to see that the surface charge density, $\sigma$, will decrease in proportion to $\theta$ :

$$
\sigma=\sigma_{0}(1-\theta)
$$

where $\sigma_{0}$ is the maximum charge density, i.e. before any adsorbate has been introduced. Solving between these two equations we Eind 


$$
\sigma=\sigma_{0} /\left(1+k[A P]_{0}\right)
$$

This equation provides the link with double layer theory, in that $\sigma_{0}$ is related to the surface potential through the Grahame equation ( $A 1$ ) in Appendix $A$, and to the experimental zeta potentials through the potential profile equation ( $A 2$ ), also in Appendix $A . K$ and $\sigma_{0}$, then, ara dur unknowns. From plots of zeta potential against aqueous concentration we can extract these constants.

\section{COMPUTATIONAL METHODS}

Initially, attempts were made to extract the binding parameters, $K$ and $\sigma_{0}$, directly from linear plots of the isotherm raw data. However, due to scatter in the data, consistent values could not be obtained and another approach was adopted. Ihis was to minimize the chi-square deviation of the experimental data from the modeled theoretical data, assuming $\sigma_{0}$ to be 1 negative charge per 65 square angstroms, a value in agreement with others in the field (21). The chi-square deviation, $\chi^{2}$, is the sum of the squared differences between experimental and theoretical zeta potentials, each point weighed according to its own experimental standard deviation squared, $\sigma_{i}^{2}$,

$$
\left.X^{2}=\sum_{i} c \zeta_{i}^{\text {theor }}-\zeta_{i}^{\exp }\right)^{2}, \sigma_{i}^{2}
$$

where the sum runs over the concentration points of a 
particular isotherm, collected as described below.

If we now turn to Appendix B, we can see this more clearly. In the figures, experimental mobilities, converted to zeta potentials with the HelmholtzSmoluchowski equation, are plotted as a function of aqueous aminopyridine concentration. The two drawn lines were theoretically computed using the Gouy-Chapman and Langmuir theories. The bottom darker curve is the screening or electrostatic cantribution to binding, corresponding to $k=$ o. The line above indicates the "tight binding", over and above the screening. The value of $K$ which reproduces this latter curve is indicated in the top left hand corner of each figure. It is this value which minimizes the chisquare deviation as defined above.

To implement the procedure, $K$ was first assumed to be zero and the theoretical zeta potential was calculated via the Gouy-Chapman equations (A1) and (A2), using $\sigma=\sigma_{0}$, and $x=2 \dot{A}$, the distance to the shear plane. The chisquare was then found. Then $K$ was increased slightly and the new value of surface potential calculated by solving between equation ( 2 ) above and ( $A 1$ ) from Appendix $A$. (The potential in equation ( 2 ) enters through the Boltzman Eactor in $\left.[A P]_{0},\right)$ There is only one value of wo that simultaneously satisfies both ( 2 ) and ( $A 1$ ). In our case, that value was found by balancing the surEace charge density with the integral of the space charge density, i.e. 
with a subroutine that found the required root of

( $\left.\sigma-\int \rho d x\right)$. (There are, of course, other ways of getting at the correct value FOr Ua). With Vo known, equation ( $A 2$ ) gives us the zeta potential and the new chi-square value could be obtained.

Then $K$ was increased Further in small steps until the value which minimized the chi-square deviation of the experimental and theoretical zeta potentials was found. Care was taken to ensure that the value of $K$ found did not correspond to a local minima. The calculations were performed on a PDP $11 / 23$ minicomputer with DIUKA1, a Fortran program developed by Dr. Smejtek. The tables Eollowing the Eigures in Appendix B show the numerical values obtained. We see the agreement is quite good.

\section{EXPERIMENTAL METHODS}

Now that the approach to the problem is outlined, we can proceed to describe the experimental determination of particle mobility.

The disodium salt of bovine brain phosphatidylserine (20 $\mathrm{mg} / \mathrm{ml}$ j was obtained from Avanti Polar Lipids (Birmingham, Alabama) and used without further treatment. A $0.5-1.0 \mathrm{ml}$ aliquat of the lipid was mixed with about 50 $\mathrm{ml}$ of analytical grade chloraform. The chloroform was distilled off in a ratary-evaporator and collected in an ice-cooled receiver, while the residue lifid formed a thin 
layer on the bottom of the round-bottomed Elask.

After an hour of sitting in the vacuum of the evaporator to ensure that all the chloroform had been removed from the lipid, the flask was shaken with $15-30 \mathrm{ml}$ of a buffered solution containing $0.1 \mathrm{M}$ tetramethylammonium chloride and $1 \mathrm{mM}$ EDIA to form a cloudy suspension of vesicles.

Ietramethylammonium chloride was the background electralyte used, since it has a very low association constant with phosphatidylserine $\left(K<.05 \mathrm{M}^{-1}(21)\right)$ and thus would simply "screen" the charged surface and not bind to it. The buffer for the pH 7.2 runs was MOPS (3-[Nmorpholinojpropanesulfonic acid), while a general-purpose borate/citrate/phosphate buffer was used for the other $\mathrm{pH}$ 's. The reason for this was that three of the compounds were only weakly ionized at neutral $\mathrm{pH}$, and so their association constants had to be obtained at $\mathrm{pH}^{\prime}$ 's were there 4 . was significant ionization. Values of $80 \%$ and $20 \%$ ionization were chosen, Cusing pKa values of 6.03, 5.98, 6.86 far 4,5-DAPM, 3-AP and 2-AP respectively), and the averaged value for $K$ was used.

A 1 molar stock solution of each aminopyridine in buffer was made up, from which $10 X$ and $100 X$ dilutians were made. The source of 4-AP, 3-AP and 3,4-DAP was Aldrich Chemical Company Inc. (Milwaukee, Wisconsin). 4,5-DAPM and 4-DMAP were from Sigma Chemical Company (St. Louis, 
Missouri), while 2-AP was Eram Reilly Chemicals. We thank Dr. Riker for providing us with many of the aminopyridines.

For each of twelve concentrations, $1 \mathrm{ml}$ of 1 ipid stock together with an appropriate volume of aminopyridine were made up to a total volume of $10 \mathrm{mls}$ with buffer. These then were used in the electrophoretic apparatus in order of increasing concentration.

The electrophoretic apparatus CRank Brothers, Mark I, Bottisham, Cambridge, U.K.J consists of a U-shaped cylindrical cell (total volume $6 \mathrm{ml}$ ), a microscope ( $\times 40$ objectivej, a d.c. power supply, two platinum electrodes and a water bath. The water bath was set to $25.0 \pm 0.5$ degrees centigrade for all measurements. An applied d.c. voltage of 20-100 $\mathrm{U}$ caused the vesicles to drift at measurable velocities. Voltage and current were monitored with a Data Precision 245 multimeter and a Dana Multimeter 4200, respectively.

In the steady state, applied valtages tended to drop slowly due to electrode polarization, i.e. small back e.m.f.'s were generated. To minimize this effect, and to average out any slight undesired drift velocities that might be occuring in the bulk solution, the applied voltage was alternately reversed in sign and the timing repeated, with the particles moving in the opposite direction. (The two velocities obtained were averaged in the data analysis: they usually agreed to within a couple of percent.) In any 
case, polarization was never allowed to exceed about $2 \%$ (i.e. no more than a $0.5 \mathrm{U}$ valtage drop over a $20.0 \mathrm{U}$ applied e.m.E.).

Particle velocity was obtained as a function of depth of microscope focus. The reason for this is that there will be a contribution to the particle velocity due to the movement of bulk liquid that the ions will carry along with them in the applied field. The bulk liquid velocity has a parabolic profile, but since the cell is closed there can be no net movement of water. It is not difficult to show with these two pieces of information that at a radius of $R / \sqrt{2}$ where $R$ is the radius of the cylinder, the bulk velocity has to be zero. This radius corresponds to the so-called stationary layer, and the vesicles will be moving solely only under their own charge at this position.

Generally at least 20 measurements were made of velocity for each concentration point. The raw data was analysed on a PDP 11 minicomputer, using ELPH4, a Fortran program developed previously in the Membrane Biophysics lab to obtain the 1 inear least squares fit for the velocity at the stationary layer--the layer in the electrophoretic cell where there is no bulk movement of liquid. The mobilities were all independent of particle size (as expected).

Eontrol measurements on free vesicles in buffer solution indicated zeta potentials comparable to published data (21). There was no evidence to suggest that the 
interlipid distance changed on adsorption of aminopyridine (which would consequently effect aur adsorption model) (26). Probably, since the aminopyridines are ions they will reside on the membrane surface where we expect pockets of potential minima (27) to be, and so not appreciably alter the interlipid distance. 
CHAPIER II I

At the end of the first chapter, the rationale behind this study was stated. Namely, two hypotheses concerning the enhanced entry of calcium into nerve terminals in the presence of aminopyridine were mentioned. One hypothesis had it that the aminopyridines were able to bind to the membrane surface and reduce the screening, the other that they were blacking potassium channels and prolanging the action potentials. In this chapter, we develop the two hypotheses further and will see that the latter is the more tenable.

First, though, we summarize the work of chapter two and notice a remarkable correlation between the binding constants and the bialogical activity of the aminopyridines.

\section{RESULTS AND DISCUSSION}

Results, in descending order of binding efficiency, are presented in Table 1. These represent the core of the thesis. The association constant of calcium cassuming $1: 1$ binding to PS), is also included. We notice all the 
aminopyridines bind more weakly than calcium.

Membrane active ion

calcium

3,4-diaminopyridine

4,5-diaminopyrimidine (*)

4-ami nopyridine

3-aminopyridine (*)

2-aminopyridine (*)

4-dimethylaminopyridine

4-aminapyridine methiodide
Association

constant $\left(\mathrm{M}^{-1}\right)$

12.1

6.5

3.8

2.6

1.8

1.6

0.5

0.2
Standard

deviation

$1 \cdot 3$

1.0

1.3

0.5

1.0

0.8

0.3

0.2

\section{IABLE I}

EXPERIMENTAL ASSOCIATION CONSTANTS $\left(M^{-1}\right)$

( (*) - AUERAGE AI TWQ PH'S )

An interesting piece of information comes to light when we look at some recent results obtained by Matsumoto and Riker (28, 29). They found the following sequence far enhancement of synaptic transmission, in the bullerog sympathetic ganglia:

$$
3,4-D A P>4-A P>3-A P>4,5-D P M>A P M I>D M A P \text {. }
$$

(See Figure 3, Ear Earmulas and abbreviations.) Aside From our analog species, 4-5-diaminopyrimidine, this order corresponds quite well with the order we have abtained above Eor the binding constants. CIn fact, the order is exact, but for DMAP and 4PMI whom we notice fall within each other's standard deviation.' 
Our sequence is alsa in agreement with Malgo et al. (5), who obtained the following ranking of enhanced evoked transmitter release,

$$
3,4-D A P>4-A P>4-A Q>3-A P>2-A P
$$

For the frog neuramusclar junction by measuring quantal content, in the absence and presence of the aminopyridines. (The quantal content is given by the ratio of mean end plate potetial (EPP) to mean miniature end-plate potential (MEPP). The MEPP would be the change in postsynaptic potential per quantum (synaptic vesicle's worthy of neurotransmitter, while the EPP would be the cumulative postsynaptic potential generated.)

We conclude that the agreement between the order of biological potency and the binding constants to PS is very strong.

THE SURFACE PDIENIIAL THEDRY

In order to understand how aminopyridines could have facilitatory effects on the calcium dependence of synaptic transmission, it will be useful to review the basic theory of the action potential, and discuss the importance of calcium in the "surface potential theory".

With the advent of the voltage clamp technique cdue primarily to Coles, which enabled the voltage-current characteristics of the nerve cell to be monitored, 
Hodgkin and Huxley (30) were able to advance their phenomenological theory of nerve transmission (in 1952). In their theory, the transmembrane current is decomposed into two components, an inward transient sodium current and a steady, outward potassium flow. The driving forces for these currents are the respective gradients of electrochemical potentials set up by active ci.e. energy consuming) transport through the membrane, which leave the membrane interior at a resting potential of -60 to $-70 \mathrm{mU}$ with respect to the exterior.

Calcium (which has been known to be an essential requirement of neural transmission since the beginning of the centuryl, when present in the external bathing solution, was soon found to simply shift the conductivityvoltage curves to the left along the voltage axis. The depolarization needed to reach firing threshold also increased. Calcium was thus found to have a stabilizing influence on the neuron.

A possible explanation for these results was advanced by Huxley in 1957 ( 31 ), who postulated the existence of a layer of Eixed negative charge on the outside surface of the membrane. The consequent negative surface potential creates an electric field that adds to the external field set up by the ionic concentration gradients. It is this total field that is sensed by the membrane components that control the conductivity of the membrane. He further 
postulated that cations should reduce the negative surface potential by binding to the surface. Hence calcium added to the external bathing solution should increase the depolarization needed to bring the Field within the membrane to a given value large enough to apen the sodium channels, i.e. they hyperpolarize the membrane. (See Figure 5, where adding cations would shift curve I to curve I I . .

In a refinement of the surface potential theory, Mclaughlin, Szabo and Eisenman ( 32 ), in 1971, clarified that cations can be effective in producing these voltage shifts by purely screening the surface charges (and not just by simply binding). In a Further application of the theory, McLaughlin and Harary (1974) (33) applied the Boltzmann relationship to the distribution of charged lipids in a lipid bilayer and suggested that if phospholipids can "Elip-flap" to redistribute themselves between inner and outer leaflets then they can effectively reduce the external field. Thus, we can in general expect an asymmetrical distribution of charged lipids across a membrane.

This membrane asymmetry in surface charge, and consequently surface potential, is indicated in Figure 5, which is taken from Hille, woodhull and Shapiro 6. 195 : (34). In this paper, they give evidence for a high density of negative charge near sodium channeis using the surface 
potential hypothesis. With reference to the figure, we note that on depolarization, the potential inside the neuron increases dramatically in the positive direction. According to their estimates then of surface potentials, the transmembrane field will then change direction, and the possibility arises that channels (in our case, we are interested in the calcium channels), can be opened and closed by changes in the transmembrane electric field.

The importance of negative surface charges on membranes seems to be gaining more recognition. Proof that the surface charges are not restricted to the axon alone comes from researchers (e.g. (35) who have obtained high negative mobilities for cerebral-cortex synaptic vesicles and synaptosomes--vesicle preparations of nerve terminals. There is nevertheless a slow acceptance of the surface potential theory in the field, perhaps because the surface potentials cannot be measured directly with electrodes--the charged region is neutralized within tens of angstroms from the surface, as we have seen. Nevertheless, the consequences of the theory, particularly with respect to the functioning of channels, may be important. 


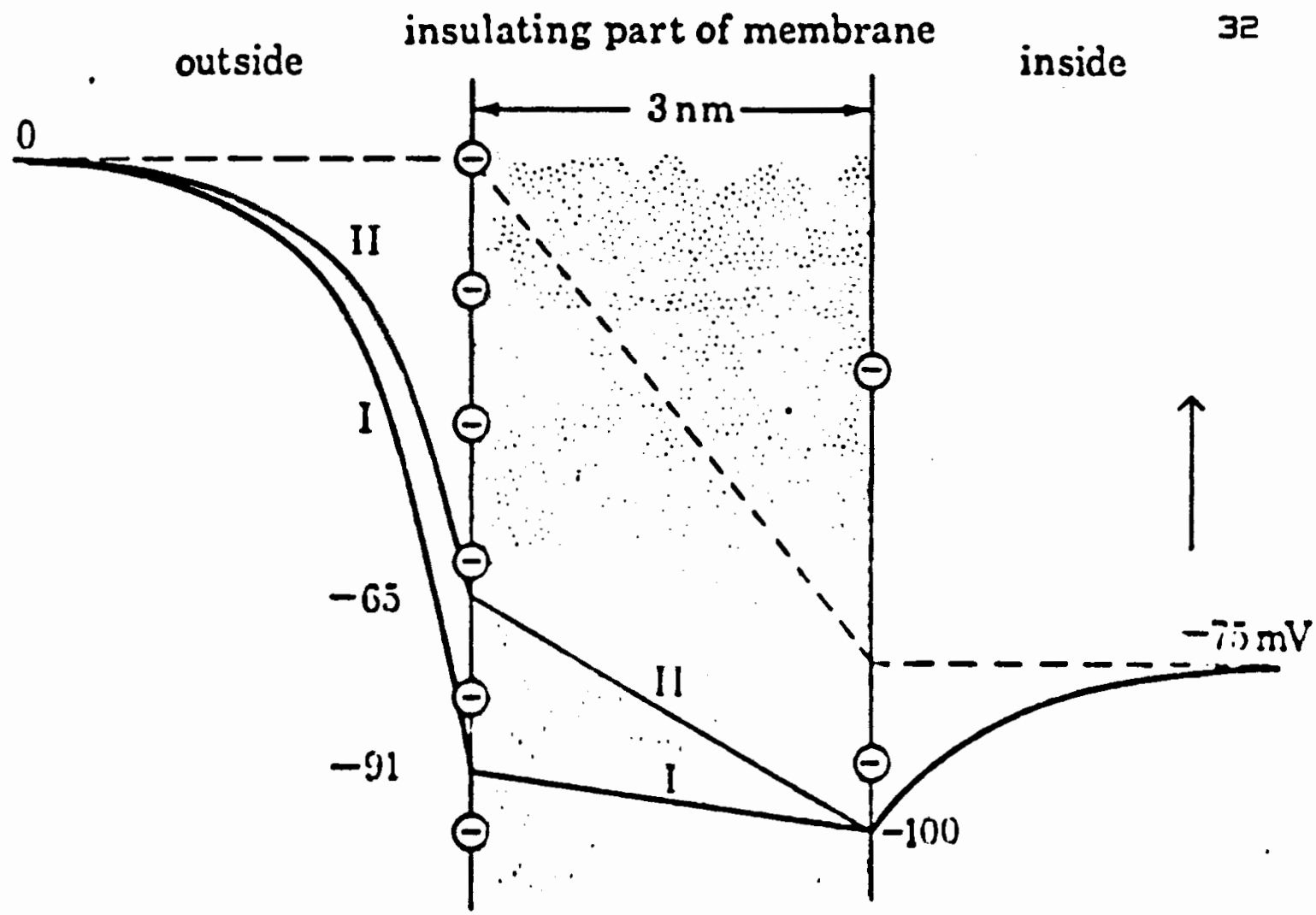

Figure 5. Postulated potential profile across an axon membrane (due to Hille et al. (34) ). The arrow shows how the potential in the inner region changes on depolarization.

A MODEL OF THE CALCIUM CHANNEL

The voltage dependence of calcium channel functioning has been measured by Ilinas and coworkers, who have also developed a mathematical model of a presynaptic calcium channel. The model gives a reasonable Fit to their voltage clamp data of squid stellate ganglion (1, 36, 37). By blocking sodium and potassium currents, they were able to monitor calcium current under various levels of controlled presynaptic depolarization.

In their model, which is of the Hodgkin-Huxley type, 
they begin with the assumption that each calcium channel is composed of $n$ subunits. Futhermore, they use a two-state kinetic model to describe each subunit. For the channel to open, each subunit, with closed state 5 , must change to its "active" state, S':

$$
S \underset{k^{\prime}}{\stackrel{k}{\vec{k}}} S^{\prime} \quad ; \quad n S^{\prime} \longrightarrow G
$$

where $k$ and $k$ 'are the forward and backward rate constants, and $G$ designates an open gate. Then the differential expression For $S^{\prime}$ will be

$$
d S^{\prime}-k s d t-k^{\prime} S^{\prime} d t
$$

and if 50 is the total number of subunits (i.e. $5=50$ $\left.S^{\prime}\right)$, then integration, with boundary condition [S'] - 0 at $t=0$, gives

$$
\left[S^{\prime}\right]=[S a]\left\{k /\left(k+k^{\prime}\right)\right\}\left[1-\exp \left[-\left(k+k^{\prime}\right) t\right\}\right]
$$

The probability of a subunit being in the $S^{\prime}$ state will be $\left[S^{\prime}\right] /[50]$, so the probability Eor a gate to be open will be $\left(\left[S^{\prime}\right] /[S a)^{n}, i . e\right.$. with all the the subunits in the S' form. Calling [Go] the total number of gates, whether open or closed, we then have for the number of open channels at any one particular time, at a particular membrane potential,

$$
[G]=[G \square]\left[\left(k /\left(k+k^{\prime}\right)\left[1-\exp \left[-\left(k+k^{\prime}\right) t\right]\right]\right]^{n}\right.
$$


Further, they assume that the rate constants are voltage dependent according to

$$
k=k_{0} \exp \left\{e z U / k_{8} T\right\} ; k^{\prime}=k_{0}^{\prime} \exp \left[e z^{\prime} U / k_{8} T\right\}
$$

where $k$ and $k^{\prime}$ do not depend on the transmembrane potential, $U$. C $U$ is taken as zero outside, so in the resting state $U$ is -60 or $-70 \mathrm{~m} U$ ). As adjustable parameters, 2 and $z^{\prime}$ represent respectively the number of charges per subunit that have to move across the membrane on changing $\mathbf{S}$ and $S^{\prime}$ into an activated transition state during the $\mathbf{S} \rightarrow \mathbf{S}^{\prime}$ transformation and its reverse. (The usual meanings apply to $e, k$ and $I . J$

The calcium flow per second, ICa, that will flow is equal to the number of open gates, [G], times the calcium Elux through an individual channel, J. The latter will be governed by the electrodiffusion equation :

$$
J--D\left(\partial c / \partial x-\operatorname{eecE} / k_{\mathrm{g}} \mathrm{I}\right)
$$

Here $D$ is the calcium diffusion constant, c the molar concentration and $E$ the electric field across the membrane. The continuity equation, $\left(\partial_{j} / \partial x+\partial_{c} / \partial t=0\right)$, then gives us

$$
\partial c / \partial t=D \partial^{2} c / \partial x^{2}-\left(2 e D / k_{B} T\right)(\partial(c E) / \partial x)
$$

In the steady state, the concentration, c, across the membrane will be constant in time and it is easy to show 
that the solution of the differential equation that satisfies $c i$ for the concentration inside the nerve terminal and co for the exteral concetration, will be

$$
J=\frac{\left\{c i-\operatorname{cosp}\left(-2 e U / k_{B} T\right)\right\}}{\left\{1-\exp \left(-2 e U / k_{B} T\right)\right\}} \frac{\operatorname{2e} D}{k_{B} I} \frac{(-U)}{L}
$$

$I$ is the thickness of the membrane and $D$ and $E$ are assumed constant along $x$. The flow decreases with membrane polarization (inside more positive) and changes sign at the calcium equilibrium potential (i.e. when $U=(k T / 2 e)$ lncei) co), approximately $-100 \mathrm{U}$, assuming $10^{-7} \mathrm{M}$ ca inside, $10^{-3} \mathrm{M}$ outside ). And so we have

$$
I C a=J A[G]
$$

where $J$ and $[G]$ are given as equations ( 3 ) and ( 4 ) and $A$ is the cross-sectional area of a single channel.

Llinas et al, obtained best Fits to their voltage clamp data on the calcium channel with $n=5, k=2 / m s e c$, $k^{\prime}=1 /$ msec, $z=1$ and $z^{\prime}=0$. Figure 6 shows their reconstruction of pre- and postsynaptic events, while Figure 7 is a schematic represent-ation of their proposed calcium channel. On the left, the channel is closed. In the middle, partially open, while on the right a conformational change in all Five monomers has been accomplished, allowing calcium to pass. 


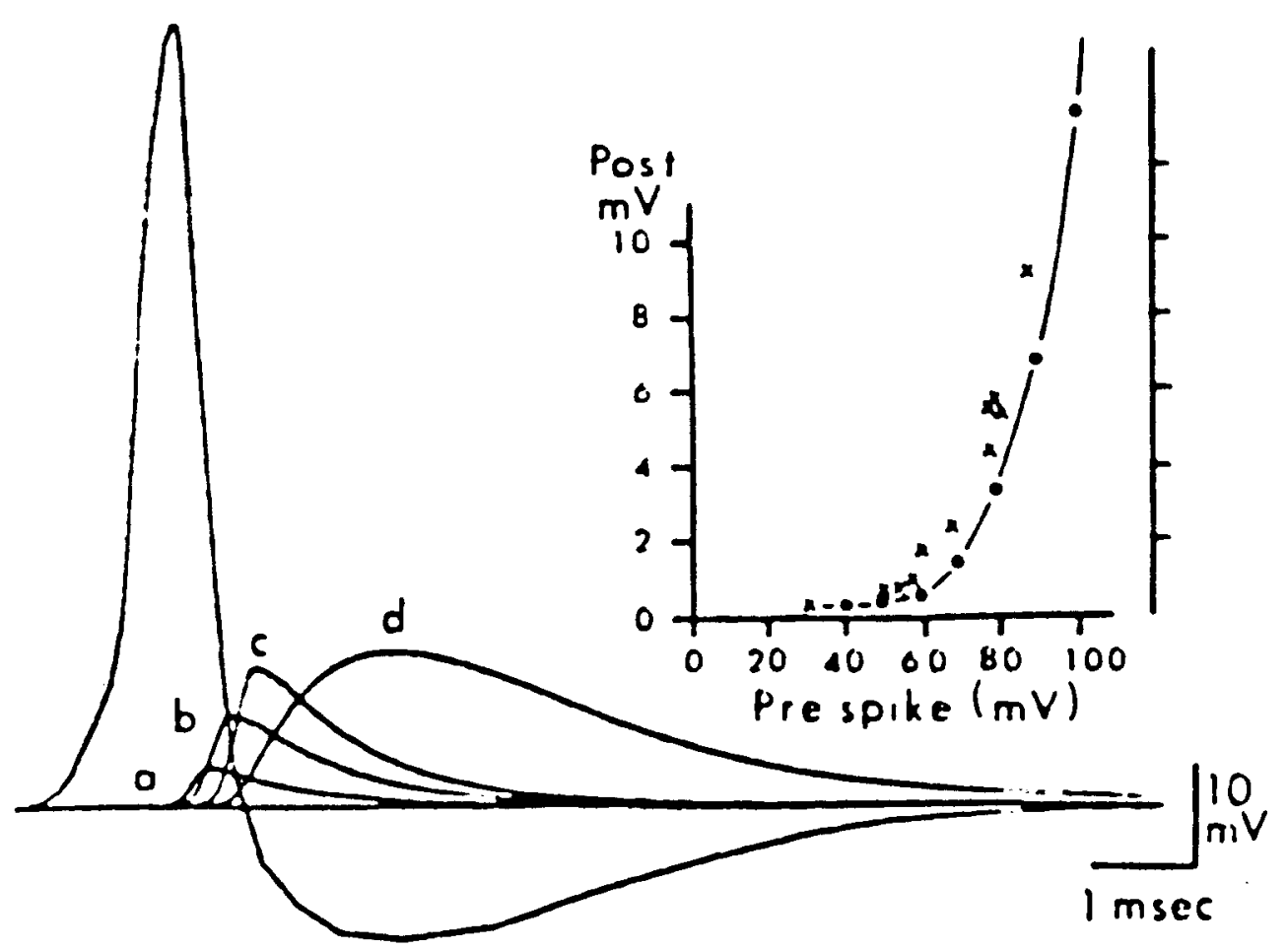

Figure B. Reconstruction of events at synaptic transmission (From LIinas ( 1 )). Iime course of (a) Ca gate formation, (b) calcium current, (c) postsynaptic current and, (d) postsynaptic potential. Inset shows experimental data $(x)$ and model data (*) For various values of presynaptic depolarizations and resultant postsynaptic potentials.

A

Outside Insias

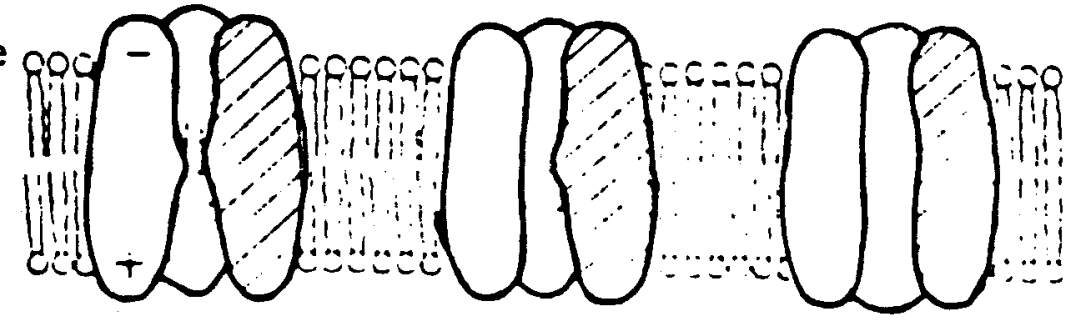

B

Inside
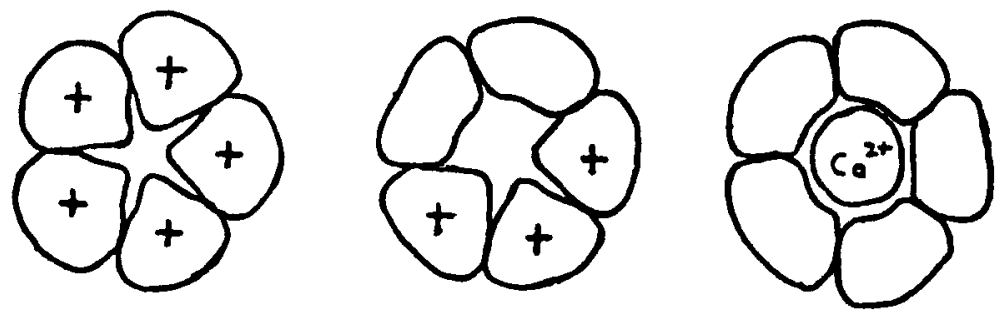

Figure 7. Hypothetical model of calcium channel in various conformations (From Llinas (1)). 
In particular, we note that the calcium current flows during the down sweep of the action potential. The magnitude of ICa is thus related to the amplitude and duration of the action potential, and modulation of these parameters will regulate the amount of neurotransmitter released. Also, because the increase in conductance, [G], comes relatively late during the action potential, it is well matched to the increased driving force for calcium entry.

The relevance of this model to aminopyridine action is clear. By blocking potassium current, the action potential is lengthened and increased calcium entry is produced. The model further predicts an increased synaptic delay--the time between the incoming action potential and the effects on the postsynaptic membrane--in the presence of aminopyridines, which is in fact observed in many experiments.

If the effect of aminopyridines is solely an $\mathrm{K}^{+}$ channels, what then is to become of the correlation of the adsorption of the aminopyridines to phosphatidylserine with the biological potency? A possible explanation is that the first step (and the rate-limiting one) of the action of the aminopyridines is their capture by the phospholipids. From the membrane surface, then, they are free to diffuse to the potassium channel proteins, with which they could interfere. Ihis "two-stage capture" process--adsorption followed by surface diffusion--is considered by Adam and 
Delbruck (38) and also by Berg and Purcell (39). The latter authors stress that efficient collection of molecules is possible simply through the three-dimensional diffusion process, if the receptor sites are evenly dispersed throughout the cell surface. Numerical calculations to verify or refute the two-stage process have not been attempted in this work. In the present case, we are dealing with diffusion through a double layer to complicate the mathematical analysis.

Alternatively, the agreement between binding constants and potency may be fortuitous--the receptor sites on the membrane and in the potassium channels may be similar. Possibly, a certain amount of "hydrophobicity" as measured by our association constants may be important to "anchor" the aminopyridines as they block $K^{+}$channels. (Thomson (12) has proposed this model from his kinetic modeling, where the aminopyridine ring rests in the membrane but the amino substituents block the channel.) Both these pictures suggest that effective aminopyridine analogs that could act as potassium channel blockers in low concentrations would have to have appropriate affinity for lipids. 
CHAPIER IU

CAN AMINOPYRIDINES DEPOLARIZE PRESYNAPIIC IERMINALS?

The original hypothesis behind the work, to test the depolarization possibility of aminopyridines, seems now difficult to support. Since the aminopyridines can be effective in low concentrations (e.g. 10 micromolar 3,4-DAP has measurable biological effects (28)), and since they have PS-binding constants of less than 10 per mole, it is difficult to argue that their binding to PS is adequate to sufficiently depolarize the membrane and produce their effects that way. For instance, assuming a Henry's law adsorption isotherm (the limiting case of a Langmuir isotherm at low concentrations), we see less than $10^{-4}$ of the lipids will be neutralized:

$$
\theta=\mathrm{K}[\mathrm{AP}]
$$

i.e.

$$
<10\left(10^{-5}\right) \text {, }
$$

where $\theta$ is the fraction of neutralized lipids. Here we have used the bulk aminopyridine concentration. Hille (34) assumes a charge density of $0.14 \mathrm{~nm}^{-2}$ for the inner membrane 
with a corresponding surface potential of $25 \mathrm{mU}$ csee figure 6). Even allowing for up to $50 \mathrm{mU}$, would give corresponding corrections (via the Boltzmann factor) to the bulk concentration of less than 10 . This does not help much. At low concentrations, surface potentials will be proportional to surface charge density. A shift of potential of several millivolts, would require, then, $\theta$ to be at least on the order of several percent. We conclude that depolarization through neutralization of negatively charged phosphatidylserine lipids cannot account for the facilitatory effects of aminopyridines.

On the other hand, this argument does not rule out the possibility of depolarization effects through binding to the lipid-bound protein. It is conceivable that these binding constants could be as high as $10^{3} \mathrm{M}^{-1}$. In fact, if surface potential could be monitored before and after application of aminopyridine, and changes on the arder of several millivolts were observed, then binding constants to proteins could be inferred. Nor is the possibility ruled out that another lipid may be responsible for tightly binding the aminopyridines, although, this would seem unlikely since for other ions, binding constants do not seem to vary drastically from lipid to lipid.

Dn the other hand, depolarization could account for the excitability effects of aminopyridines. The fact that these effects are not universally reported could reflect 
different surface charges on different preparations. Matthews and wickelgren (40) calculated a depolarizetion of 5-7 mU for $0.3 \mathrm{mM}$ guanidine and measured a decreased threshold potential from 14 to $B \mathrm{mU}$. CGuanidine is another Facilitatory quaternary compound, which has similar actions to the aminopyridines.) However, the calculation of this depolarization value involved measuring quantal content while varying the external potassium concentration. The shift in potassium concentration, in the presence of guanidine, to reproduce similar quantal contents without guanidine was then used, via the Goldman-Kodgkin-Katz equation (see, for instance, Benedek (24), page 3-222) to calculate the depolarization. But since altering external potassium levels will most likely effect the kinetics of the potassium channels, it is unclear that this value is reliable. Also, these authors assumed the guanidine bound to the outside of the membrane. It seems from our discussion that the binding would have to be to the internal surface to depolarize the cell.

\section{CDNCLUSIDN}

The binding constants for a series of aminopyridines to phosphatidylserine vesicles were determined. The order of binding was approximately the same as the order of potency in enhancing synaptic transmission in the presence of reduced calcium concentrations and in normal calcium 
concentrations. However, the constants were too low to argue that the aminopyridines could cause their effect by depolarizing the presynaptic membrane by binding to phosphatidylserine at the concentration levels used in the biological experiments. However, they may be able to depolarize it in higher concentrations if there is a substantial difference in surface potential between the outside and inside of the membrane.

A simple model of a calcium channel was discussed that could explain most of the observations of aminopyridine actions. The hypothesis was proposed that the agreement in the order of aminopyridines between their potency in synapses and their association constants with phosphatidylserine membranes may indicate that adsorption to the membrane is the first and rate-limiting step. An alternative possibility would be that the aminopyridines are anchored in the membrane when they are in position to block potassium channels. At the present time it is neither possible to distinguish between these two possibilities nor to verify either of them. Finally, although it cannot be ruled out that the similarities in the sequences are purely fortuitous, we close by hoping that this correlation will find a satisfactory resolution in the future and that the details of aminopyridine action will be made clear. 


\section{REFERENCES}

1. LLINAS R.R. and J.E. HEUSER. 1977. DepolarizationRelease Coupling Systems in Neurons. Neurosciences Research Program Bulletin 15: 555-687.

2. KATZ B. and R. MILEDI. 1967. The release of acetylcholine from nerve endings by graded electrical impulses. Proceedings of the Royal Society B 167: 23-38.

3. MATSUMOTO M. and $W . K$. RIKER. 1983. Synaptic transmission in low extracellular celcium is preserved by 3,4-diaminopyridine. Journal of Pharmacology and Experimental Therapeutics 228: 16-21.

4. SMEJIEK P., W.K. RIKER, A. DXYZDGLDU, C. WRIGHT and M. BENNETI. 1985. Adsorption of aminopyridines to phosphatidylserine membranes. Biophysical Journal 49: $339 a$.

5. MOLGD J., M. LEMEIGNAN and P. LECHAT. 1985. Increase in evoked transmitter release from motor nerve terminals by some amino N-heterocyclic compounds. I Comparetive experimental activities and extracellular pH-dependence. European Journal of Medicinal Chemistry--Chim. Ther. 20, No.2: 149-153.

6. DOHRN M. 1924. Pharmakologie einiger Pyridinderiate. Arch. exp. Path. Pharmak. 105: 10-11.

7. PELhate M., B. HUE, Y. PICHDN and J. CHANELET. 1974. Action de la 4-aminopyridine sur la membrane de 1 'axone isole d'insecte. Comptes Rendus de l' Academie des Sciences (Paris), Serie D 278 : 28072809.

B. LECHAT P., S. THESLEFF and W.C. BDWMAN, eds. 1982. Aminopyridines and similarly Acting Drugs: Effects on 
Nerves, Muscles and Synapses. Advances in the Biosciences, volume 35, Permagon Press, Dxford.

9. GLQUER W.E. 1982. The aminopyridines. General Pharmacology 13: 259-285.

10. THESLEFF 5. 1980. Aminopyridines and synaptic transmission. Neuroscience 2 : 1413-1419.

11. ULBRICHI $W$. and WAGNER H.-H. 1976. Block of potassium channels of the nodal membrane by 4-aminopyridine and its partial removal on depolarization. PEluggers Archiv 367 : 77-87.

12. THOMPSON 5. 1982. Aminopyridine block of transient potassium current. Journal of General Physiology 80: 1-18.

13. KIM Y.I., M. M. GOLDNER and D. B. SANDERS. 1980. Facilitatory effects of 4-aminopyridine on neuromuscular transmission in disease states. Muscle and Nerve 3: 112-119.

14. YEH J.Z., G.S. OXFORD, C.H. WU and I. NARAHASHI. 1976. Interactions of aminopyridines with potassium channels of squid axon membranes. Biophysical Journal 16: 77-81.

15. BOWMAN W.C., A.L. HARUEY AND I.G. MARSHALL. 1977. The actions of aminopyridines on avian muscle. Naunyn-Schmiedeberg's Archives of Pharmacology 297: 99-103.

16. MARSHALL I.G., J.J. LAMBERT AND N.N. DURAND. 1979. Inhibition of aminopyridine-induced contractile activity in skeletal muscle by tetrodotaxin and by magnesium. European Journal of Pharmacology 54: 914 .

17. AUDIBERT-BENOIT M.-L. 1975. Effets de la 4aminopyridine sur les proprietes membranaires de la Fibre musculaire squelettique de Grenouilie. Journal de Physiologie (Paris) 72: A1.

18. MAETANI T., Y. SHIBA, Y. MUNEDKA and Y. KANND. 1979. Effects of $Z n$, $U D$ and 4-aminopyridine on twitch contraction and action potential in the mouse skeletal muscles. Hiroshima Journal of Medicinal Sciences 28: 43-48.

19. KIM Y.I., M.M. GOLDNER and D. B. SANDERS. 1980. Facilitatory effects of 4 -aminopyridine on normal 
neuromuscular transmission. Muscle \& Nerve 3: 105111 .

20. DUERBEEK J.TH.G and P.H. WIERSEMA. 1967. The interpretation of electrophoretric mobilities. In Electrophoresis, Theory, Methods and applications. M. Bier, ed., Academic Press, New York and London. 2 : $1-52$.

21. EISENBERG M., I. GRESALFI, T. RICCID and $\mathbf{s}$. MCLAUGHLIN. 1981. Adsorption of monovalent cations to bilayers containing phosphatidylserine. Journal of General Physiology 77: 445-43.

22. QUERBEEK J.TH.G and J. LIJKLEMA. 1959. Electric potentials in colloidal systems. In Electrophoresis, Iheory, Methods, Methods and applications. M. Bier, ed., Academic Press, New York and London. 1:1-33.

23. DAUIES J.I. and E.K. RIDEAL. 1963. Interfacial

Phenomena. 2nd edition. Academic Press, New York. Pages 140-144.

24. BENEDEK G.B. and F.M.H. UILLARS. 1979. Physics with illustrative examples From medicine and biology. Addison-Wesley Pub. Co., Reading, Massachusetts. Chapter 3 .

25. RICE S.A. and M. NAGASAWA. 1961. Polyelectrolyte Solutions. Academic Press, New York and London. Chapter 4 .

26. IAKED M, B.A. SINHA and G.E. REMPFER. 1985. AdsOrption of slightly soluble compounds to lipid monolayers. Journal of Colloid and Interface Science 101: 384391 .

27. FLEWELLING R.F, and W.L. HUBBELL. 1986. The membrane dipole potential in a total membrane potential model: applications to hydrophobic ion interactions with membranes. Biophysical Journal 49: 541-552.

2B. MATSUMOTD M. AND W.K. RIKER. 1984. EFfect of several aminopyridines and analogs on the calcium dependence of synaptic transmission. Journal of Pharmacology and Experimental Therapeutics 228: 573-578.

29. RIKER W.K., M. MATSUMDID and K. IAKASHIMA. 1985. 
Synaptic Facilitation by 3-aminopyridine and its antagonism by verapamil and diltiazem. Journal of Pharmacology and Experimental Therapeutics 235: 431435 .

30. HODGKIN A.I. and A.F. HUXLEY. 1952. A quantitative description of membrane current and its application to conduction and excitation in nerve. Journal of Physiology 117 : 500-544.

31. HUXLEY A.F. in B. FRANKENHAEUSER and A.L. HODGKIN. 1957. The action of calcium on the electrical properties of squid axons. Journal of Physiology 137: 218-244.

32. Mclaughlin S.G.A., G. SZABD and G. Eisenman. 1971. Divalent ions and the surface potential of charged phospholipid membranes. Journal of General Physiology 58: 667-687.

33. MCLAUGHLiN S. and H. HARARY. 1974. Phospholipid ElipFlop and the distribution of surface charges in axcitable membranes. Biophysical Journal 14: 20020B.

34. HILLE B., A.M. WODDHULL and B.I. SHAPIRO. 1975. Negative surface charge near sodium channels of nerve: divalent ions, monovalent ions and pH. Philosophical Iransactions of the Royal Society of London B 270: 301-318.

35. Mclaughlin J., K.R. CASE and H.B. BDSMANN. 1973.

Electrokinetic properties of isolated cerebral-cortex synaptic vesicles. Biochemical Journal 136: 919-926.

36. LLINAS R., I.Z. SIEINBERG and K. WALIDN. 1976. Presynaptic calcium currents and their relation to synaptic transmission: Uoltage clamp study in squid giant synapse and theoretical model for the calcium gate. Proceedings of the National Academy of Sciences 73: 2918-2922.

37. LLINAS R., M. SUGIMORI and 5. M. SIMON. 1982. Iransmission by presynaptic spike-like depolarization in the squid giant synapse. Proceedings of the National Academy Sciences 79: 2415-2419.

38. ADAM G. and M. DELBRUCK. 1968. Reduction of dimensionality in biological diffusion processes. In Structural Chemistry and Molecular Biology. A. Rich and N. Davidson, editors. W.H. Freeman and Company, Publishers, San Francisco, Calif. 198-215. 
39. BERG H.C. and E.M. Purcell. 1977. Physics of chemoreception. Biophysical Journal 20: 193-219.

40. MATIHEWS G. and W.Q. WICKELGREN. 1977. EfFects of guanidine on transmitter release and neuronal excitability. Journal of Physiology 266: 69-89.

41. MCLAUGHLIN 5. 1977. Electrostatic potentials at membrane-solution interfaces. Current Iopics in Membrane Iransport 9: 79-144.

42. JONES M.N. 1975. Biological Interfaces: an introduction to the surface and colloid science of biachemical and biological systems. Elsevier Scientic Pub. Co., New York.

43. ABRAHAM-SHRAUNER B. 1975. Generalized Gouy-Chapman potentials of charged phospholipid membranes with divalent cations. Journal of Mathematical Biology 2 : 333-339. Note also: Erratum for .... Journal of Mathematical Biology 4: 201.

44. BENTZ J, and S. NIR. 1980. Competition between mono-, di-, and trivalent cations. Bulletin of Mathematical Biology 42: 191-220.

45. ROBINSON R.A. and R.H. STOKES. 1968. Electrolyte Solutions. 2nd edition(revised). Butterworth, London. Chapter 4.

45. UERWEY E.J.W. and J.TH.G. DUERBEEK. 1948. Theary of the Stability of Lyophobic Clouds. Elsevier, Amsterdam and New York. Page 23.

47. NELSON A.P. and D.A. MCQUARRIE. 1975. The effect of discrete charges on the electrical properties of a membrane. International Journal of Theoretical Biology 55: 13-27.

48. BIKERMAN J.J. 1970. Physical Surfaces. Academic Press, New York and London. Page 375.

49. LUZAR A., S. SUEIINA and B. 2EKS. 1984. Polarization of water at the solid-liquid interface and the distributions of ions close to the phosphalipid membrane. Bioelectrochemistry and Bioenergetics 13: 473-4B4 (a section of the Journal of Electroanalytical Chemistrys. 
APPENDIX A

Gouy-Chapman Iheory

It was Helmholtz (1879) who first developed a mathematical theory concerning the electrical double layer --the combination of a charged surface and a layer of oppositely charged ions attracted to it in a solution. Gouy (1909) and Chapman (1913) proposed the idea of a "diffuse double layer", whereby thermal motion tends to disrupt the Fixed Helmholtz double layer. Stern (1924) combined the features of both the sharp and the diffuse double layers by postulating a thin layer of bound counterians beyond which lay a diffuse layer. The theory, which applies anly to Elat surfaces, is essentially unchanged today.

The mathematical Eramework of the Gouy-Chapman theory is the combination of the Poisson equation to account for the electrostatic attraction to the charged surface, and the Boltzmann equation to take into account the thermal motion of the ions ( 41,42$)$. (These two Features also form the basis of modern electrolyte theory, and are also used 
in plasma and semiconductor physics.)

The potential, $U$, in the space charge region adjacent to the charged surface will satisfy the Poisson equation in the equilibrium case (MKS units are used throughout),

$$
d^{2} u / d x^{2}--\rho / \epsilon
$$

where $\rho$ is the Eree charge density, $\epsilon=\epsilon_{0} \epsilon_{m}$ is the product of the vacuum permittivity and the dielectric constant respectively, and $x$ represents the distance normal to the charged surface. In thermal equilibrium, with ions of charge $q_{i}$ and concentration (per cubic meter) $c_{i}$, the charge density will be, with appropriate Boltzmann Eactors (e, $k_{0}$ and I have their previous meanings):

$$
\rho=\sum_{i} c_{i} q_{i} \exp \left(-q_{i} U / k_{b} T\right)
$$

Combining, we then have,

$$
E d^{2} U / d x^{2}=-\sum_{i} c_{i} q_{i} \exp \left(-q_{i} U / k_{B} T\right)
$$

The "Poisson-Boltzmann" equation can be solved with the aid of the substitution, $d^{2} U / d x^{2}=1 / 2 d / d U(d U / d x)$, whence,

$$
1 / 2 \in(d U / d x)^{2}=-\int d U \sum_{i} c_{i} a_{i} \exp \left(-q_{i} U / k_{0} T\right)
$$

After performing the integral and, with the boundary condition that $d U / d x=0$ when $U=0$ (at $x-\infty)$, we get 


$$
\epsilon(d U / d x)^{2}=2 k_{B} T \sum_{i} c_{i}\left(\exp \left(-q_{i} U / k_{B} T\right)-1\right]
$$

Since at the surface, $\sigma=-\epsilon d U / d x$, we obtain from the above equation, the Grahame equation, which relates the surface charge density, $\sigma$, to the surface potential, Uo,

$$
\sigma=\left(v_{0} / 1 v_{0}\right)\left[2 k_{B} I \in\right]^{\frac{1}{2}}\left[\sum_{i} c_{i}\left(\exp \left(-q_{i} v_{0} / k_{B} I\right)-1\right)\right]^{\frac{1}{2}}
$$

Io proceed further, we restrict ourselves to a z:z electralyte, concentration $c$. There will be only two terms in the sum and the Grahame equation reduces to

$$
\sigma=\left(V_{0} / V_{a}\right)\left(\theta k_{B} I c \in\right)^{\frac{1}{2}} \sinh \left(z e V_{0} / 2 k_{B} T\right)
$$

A second integration of the Poisson-Boltzmann equation can be performed, using the relation $\int d p / s i n h p=1 n$ [tanh $(p / 2)]$, leaving

$$
\begin{aligned}
\text { In }\left[\tanh \left(z e U_{0} / 4 k_{b} I\right) / \tanh \right. & \left.\left(z e U / 4 k_{b} T\right)\right]= \\
& x /\left[\left(\epsilon k_{B} I\right) /\left(2 \theta^{2} z^{2} c\right)\right]^{\frac{1}{2}}
\end{aligned}
$$

or, defining the Debye length, $\lambda_{0}-\left[\left(\epsilon k_{b} T\right) /\left(2 e^{2} z^{2} c\right)\right]^{\frac{1}{2}}$,

$$
\tanh \left(z e U / 4 k_{B} T\right)=\tanh \left(z e U_{0} / 4 k_{B} T\right) \exp \left(-x / \lambda_{0}\right) \text { (AZ). }
$$

For zeU/k $I \ll 1$, (i.e. $U \ll 25 \mathrm{mU}$, for a monovalent salt at room temperature), (A2) reduces to the purely exponential decay,

$$
u=v_{0} \exp \left(-x / \lambda_{0}\right)
$$

with a decay length, $\lambda_{0}$, (Debye screening length) of, for 
instance, 10 angstroms for a decimolar univalent electrolyte at $25^{\circ} \mathrm{C}$. The Debye length gives the formal expression for our previously ill-defined "thickness" of the double layer in chapter two. In the case of small potentials, a simple interpretion is possible. The Debye length is the distance for the potential to drop to $1 / e$ of its surface value.

Abraham-5chrauner has also obtained an analytical solution to the planar Poisson-Boltzmann equation for the case of a 2:1 electrolyte (43). Bentz and Nir ( 44 ) have generalized her equation to accomodate any combination of mono and divalent ions. Their equation (A11) was used in determining the binding constant for calcium. (In our case, because we also had in some of our solutions small amounts of trivalent buffer, a numerical program was also written to solve the Poisson-Boltzmann equation for any combination of ions of any charge. However, the program was slow and proved not to be necessary.)

Before leaving the electrical double layer, we nots some of the approximations inherent in the theory. First we mention that many important physicists and physical chemists (Fowler, Guggenheim, Onsager, Kramers, Kirkwood and Casimir) have worked on the problem of an inconsistency in the Poisson-Boltzmann equation, which arises when the average potential in the Poisson equation is equated with the "potential of mean Force" in the Boltzmann relation. 
(See Dverbeek and Lijklema (2己) for original references, also (45).) This is so because $q_{i} U(x)$ ought to be the work done to bring ion $i$ to position $x$, but when the ion is at $x$, it will influence the distribution of ions in its neighborhood, so that the potential at $\times$ will deviate from the mean potential $U(x)$. Verwey and Dverbeek (4E) state that "for large $U$, the determination of the mean value of c From $U$ is strictly speaking not allowed." However, the general consensus is that in the Full non-linear PoissonBoltzmann equation the error introduced will be small when the flat surface bears many charges and the ionic concentrations do not rise to values at which deviations From the Debye-Huckel laws become considerable.

Also implicit in the Gouy-Chapman theory is the neglect of discrete charge effects--we deal only with "smeared" charge densites (47). Furthermore, the ions are treated as point charges. Ihus we ignore that they have a Finite volume and an individual polarizability (and perhaps a permanent dipole moment), and that they will displace an equal volume of solvent molecules which will also have a polarizability and dipole moment ( 48 ).

Polarization effects at the interface ca.g. From restrictions on solvent orientation ( 49 ) or from the lipid head groups (27)) are also ignored. Finally, the dielectric constant is assumed not to vary as the interface is approached (27, 49). However, despite its shortcomings 
and the liklihood that some of the approximations compensate for each other, we have found that the basic Gouy-Chapman-Stern theory gives an adequate description to our data. 


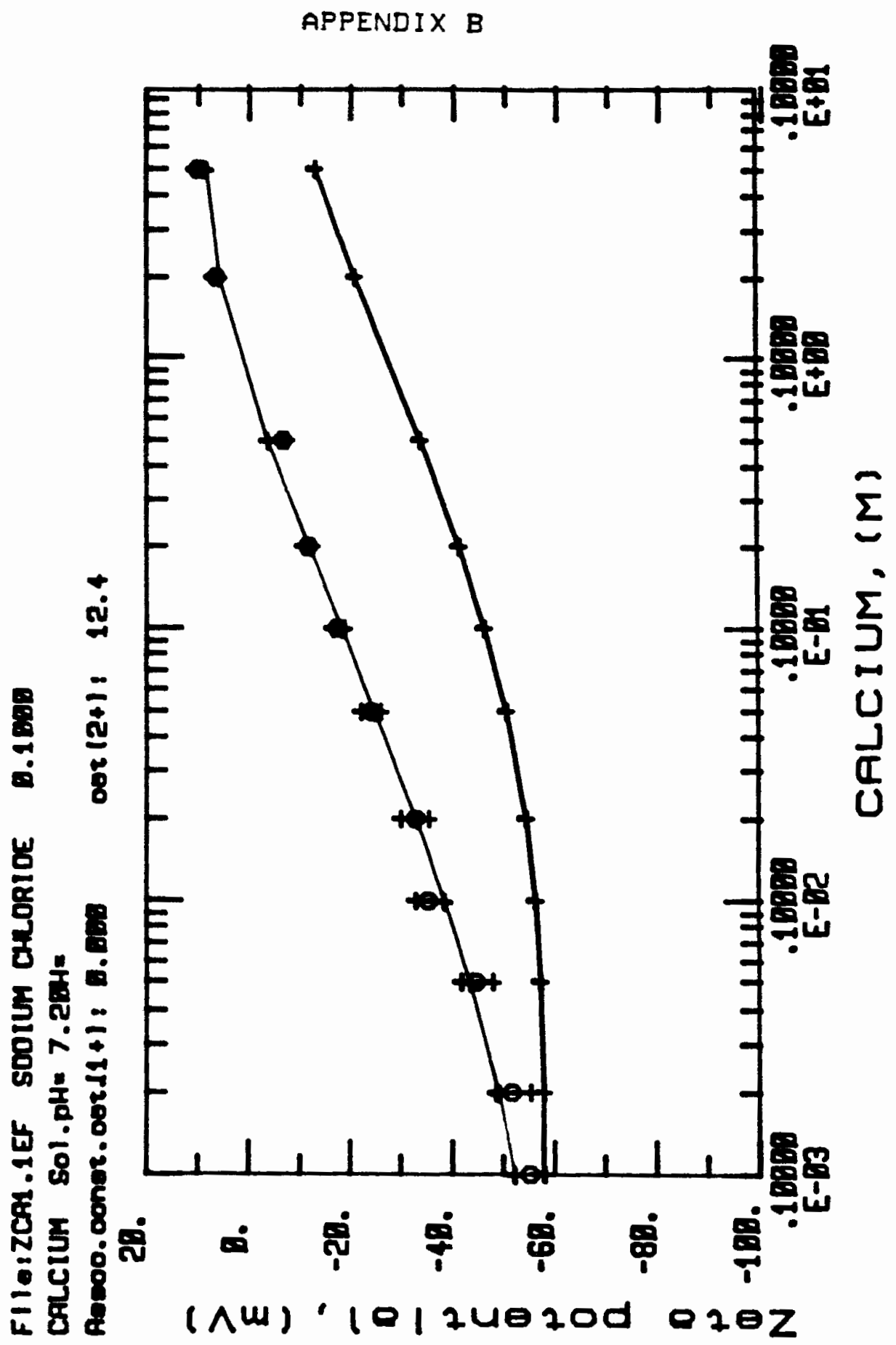

Figure B. Adsorption isotherm for calcium. 


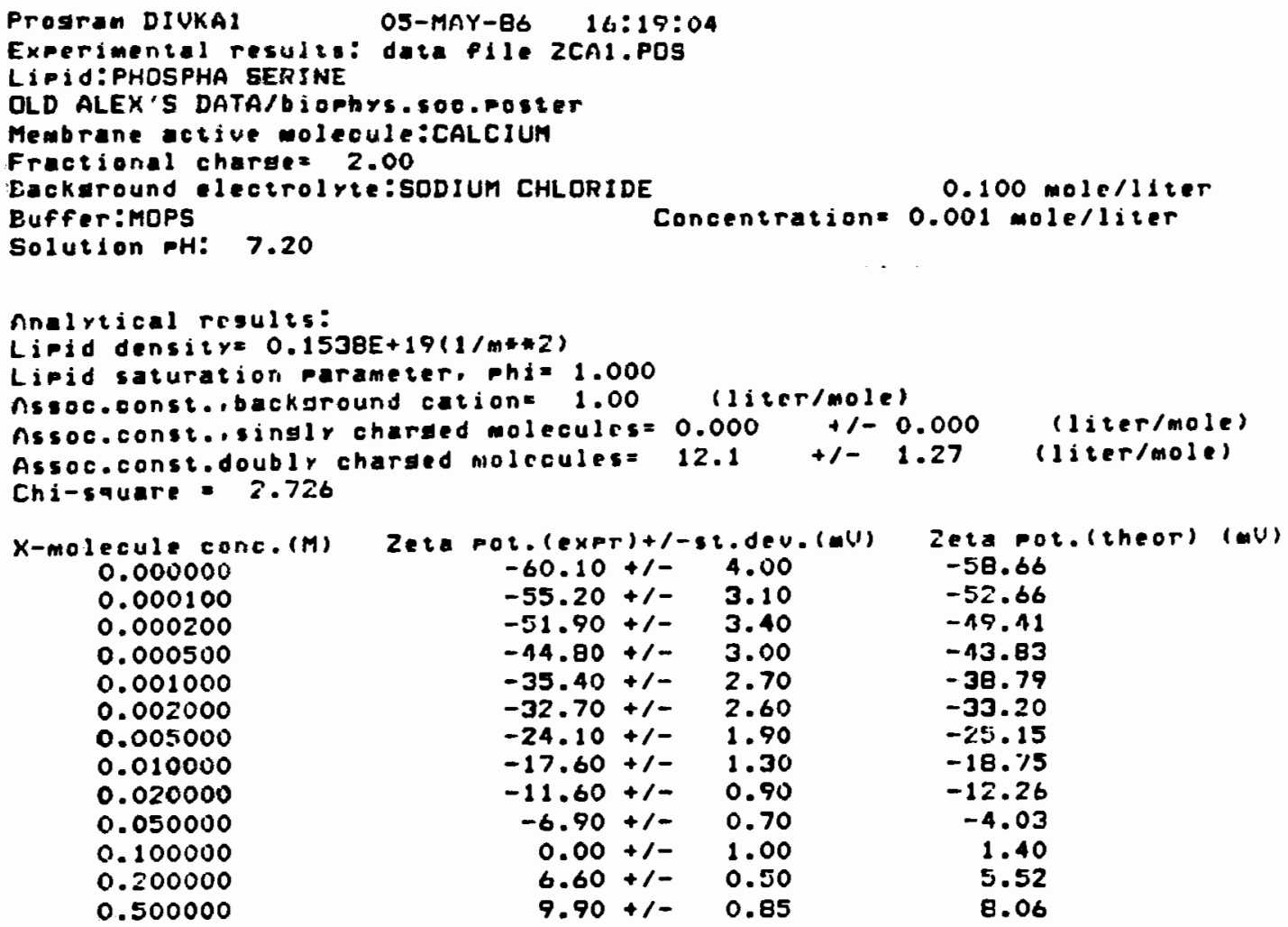




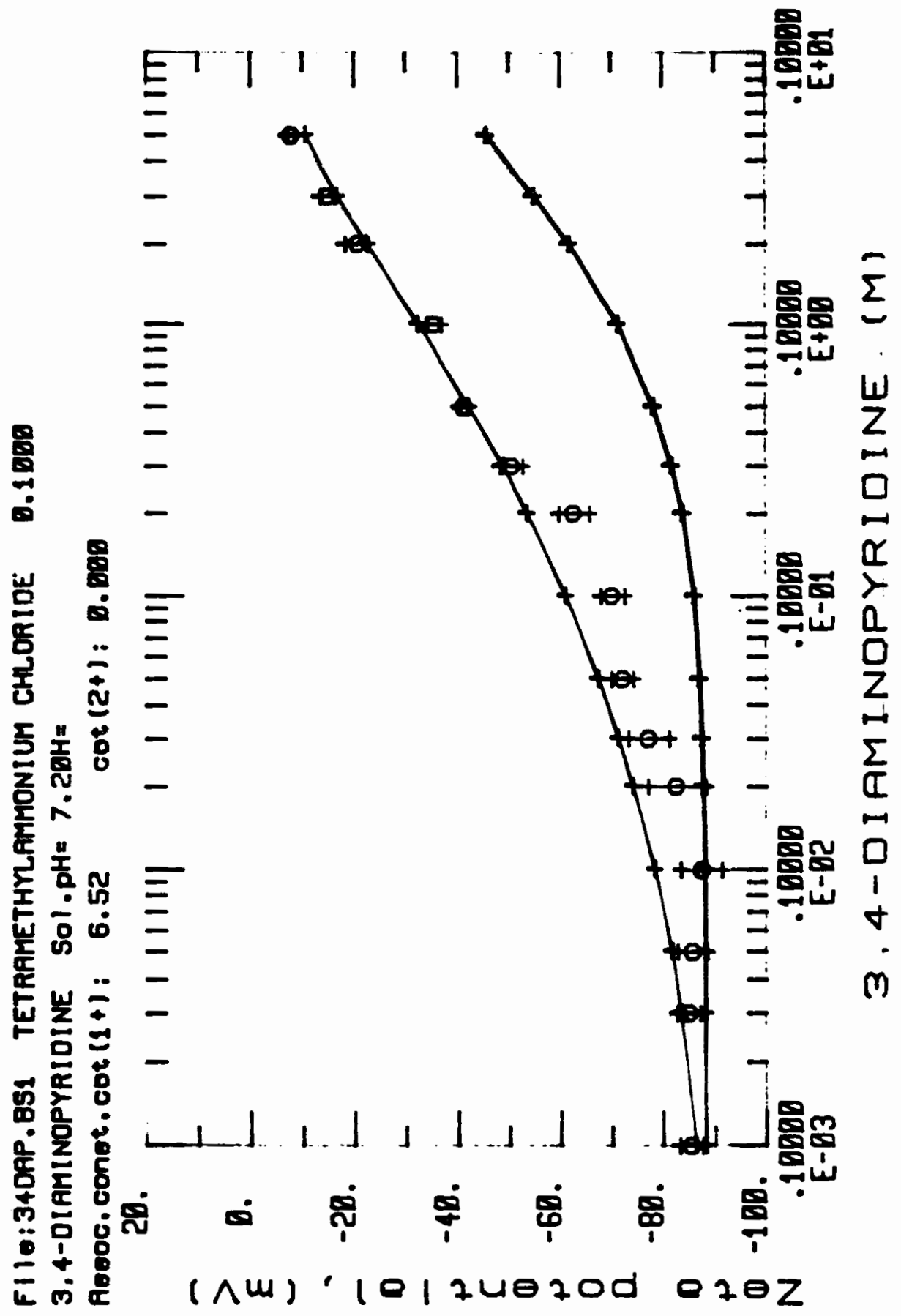

Figure 9. Adsorption isotherm far 3,4-diaminopyridine. 


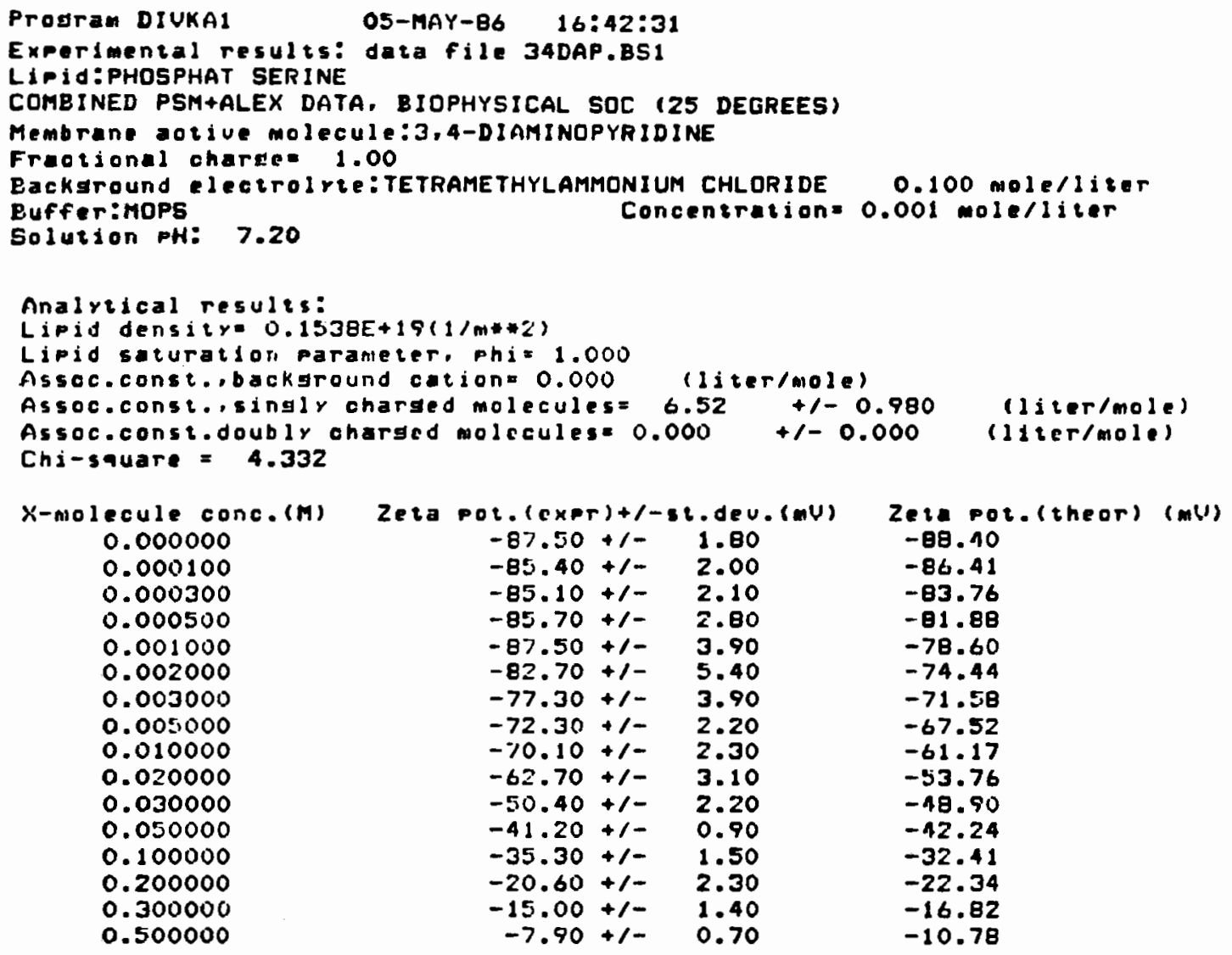




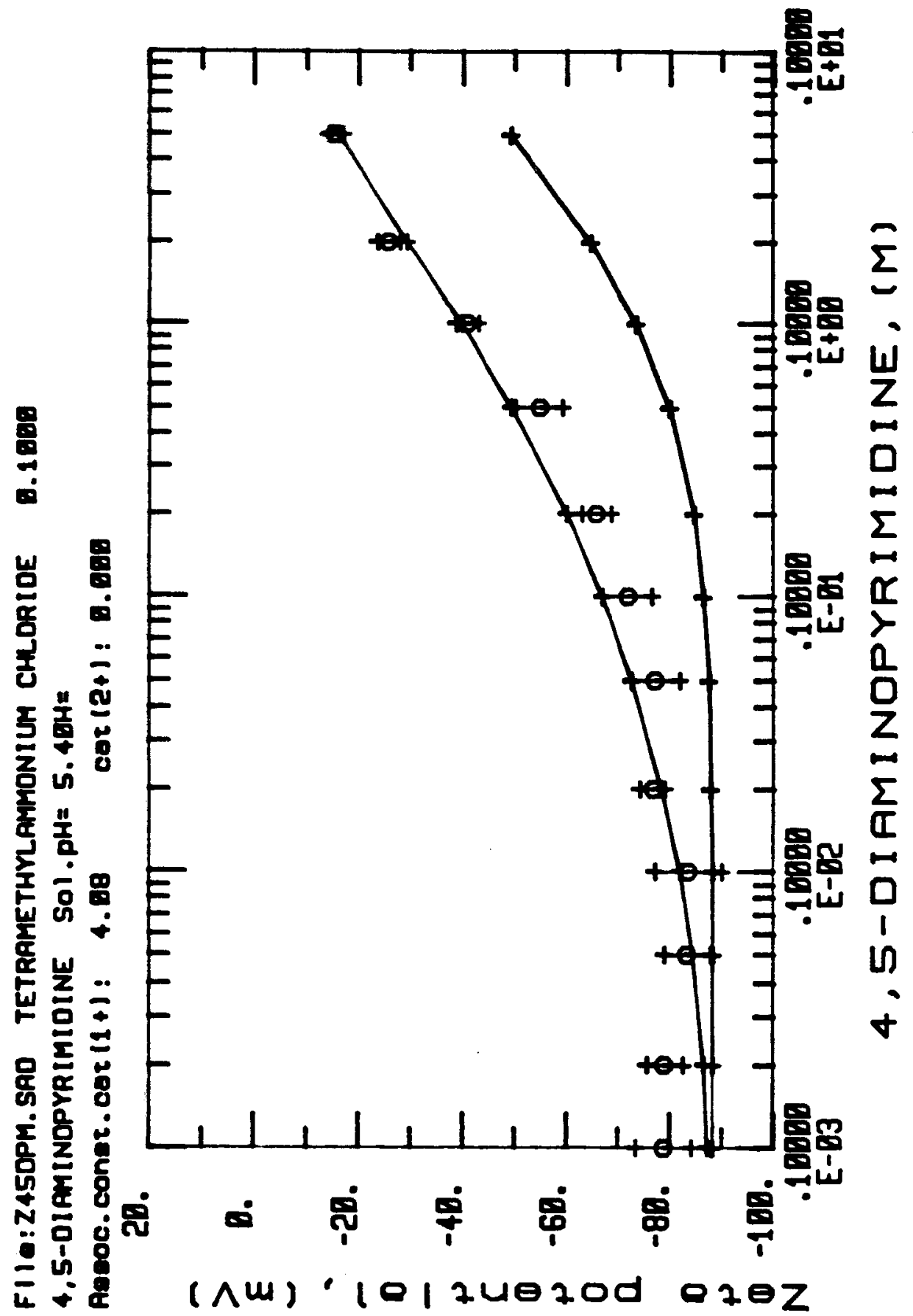

Figure 10. Adsorption isotherm for

4,5-diaminopyridine (pH 5.4). 


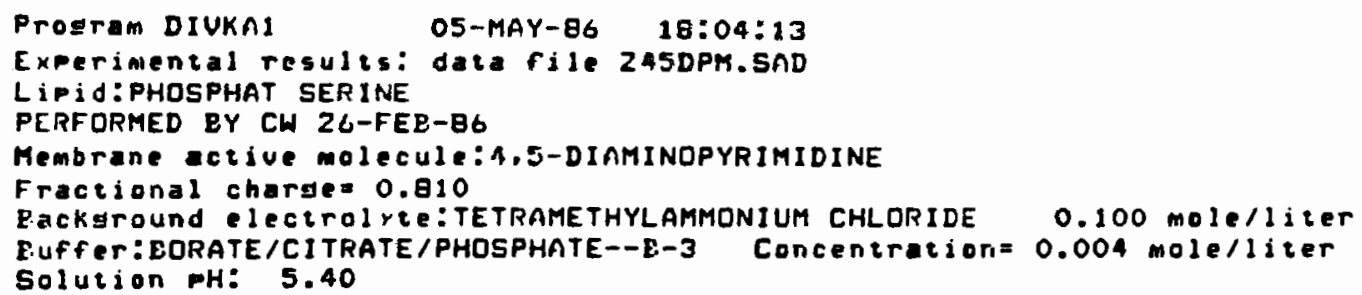

\begin{tabular}{|c|c|c|c|}
\hline & $\begin{array}{l}-81.40 \\
-79.00 \\
-79.00 \\
-83.40 \\
-83.60 \\
-76.70 \\
-77.10 \\
-71.70 \\
-65.70 \\
-54.50 \\
-40.60 \\
-25.60 \\
-15.50\end{array}$ & $\begin{array}{l}+1- \\
+1- \\
+1- \\
+1- \\
+1- \\
+1- \\
+1- \\
+1- \\
+1- \\
+1- \\
+1- \\
+1- \\
+1-\end{array}$ & $\begin{array}{l}5.30 \\
5.30 \\
3.40 \\
4.60 \\
6.50 \\
2.20 \\
4.70 \\
4.90 \\
2.90 \\
4.60 \\
2.20 \\
2.10 \\
1.20\end{array}$ \\
\hline
\end{tabular}

zeta pot. (theor) (mU) $-88.40$

$-87.28$

$-86.35$

$-84.72$

$-81.72$

$-78.35$

$-72.47$

$-66.85$

$-60.11$

$-19.28$

$-39.65$

$-29.22$

$-16.02$ 


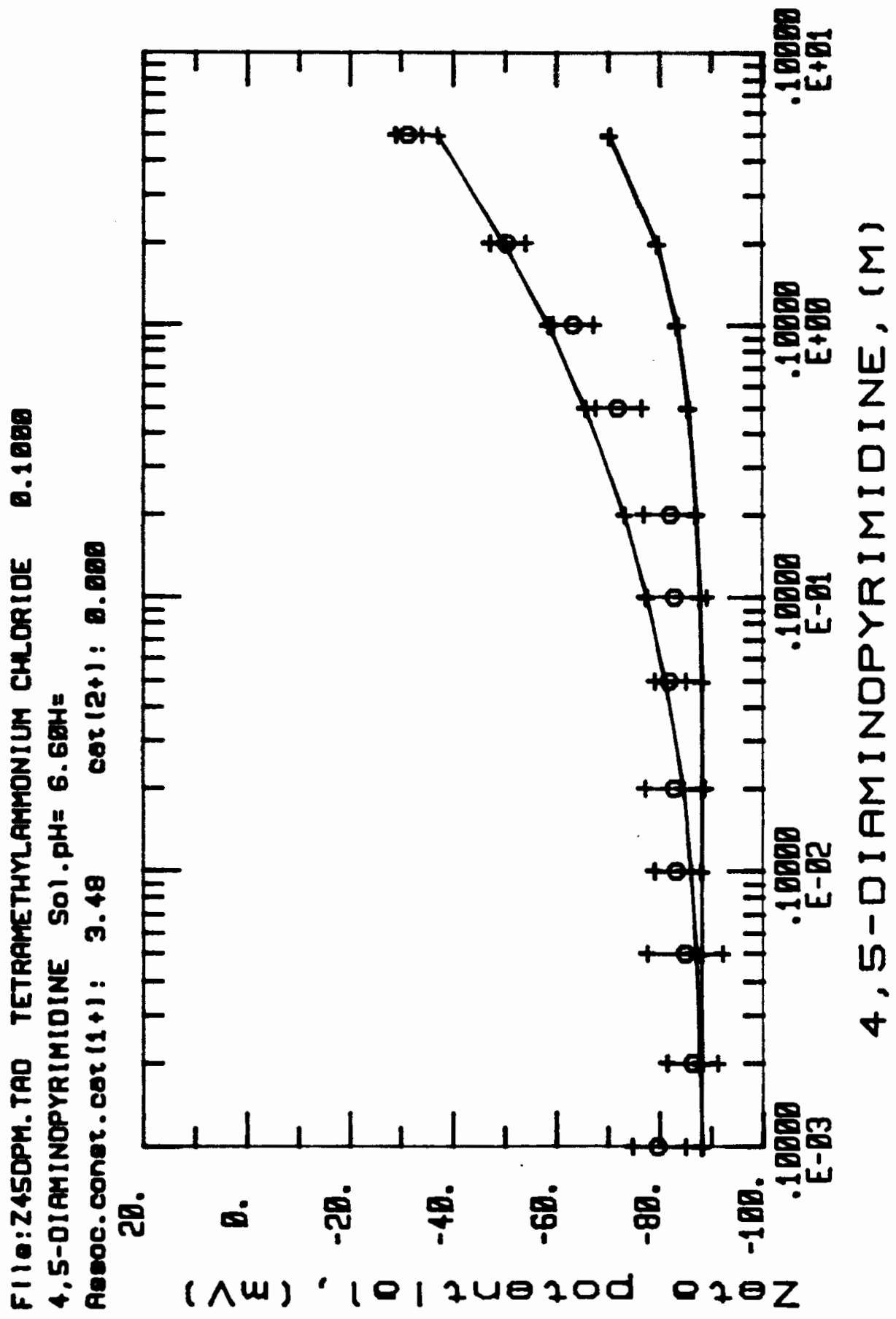

Figure 11. Adsorption isotherm For 4,5-diaminopyrimidine (pH 6.6). 


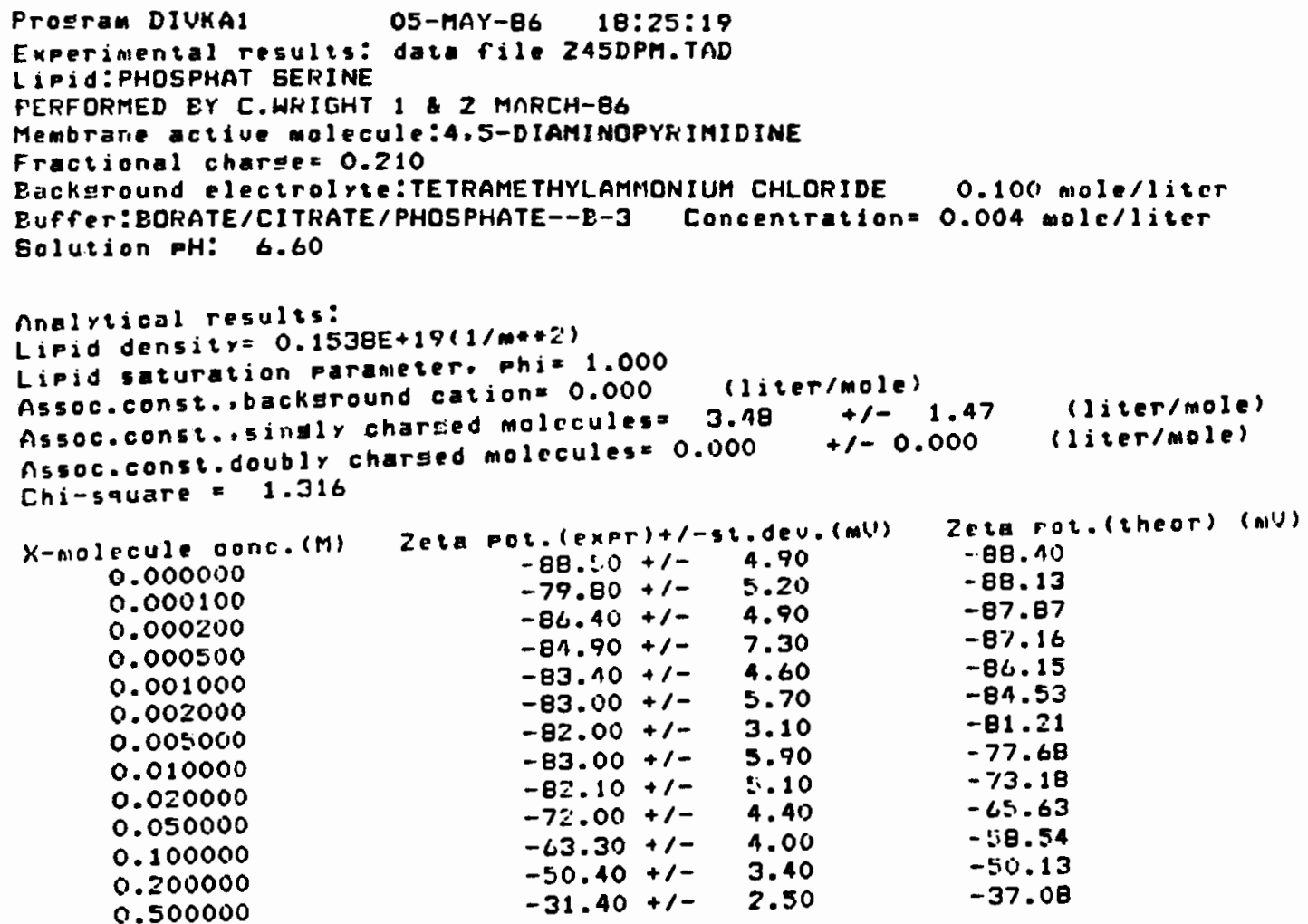


62

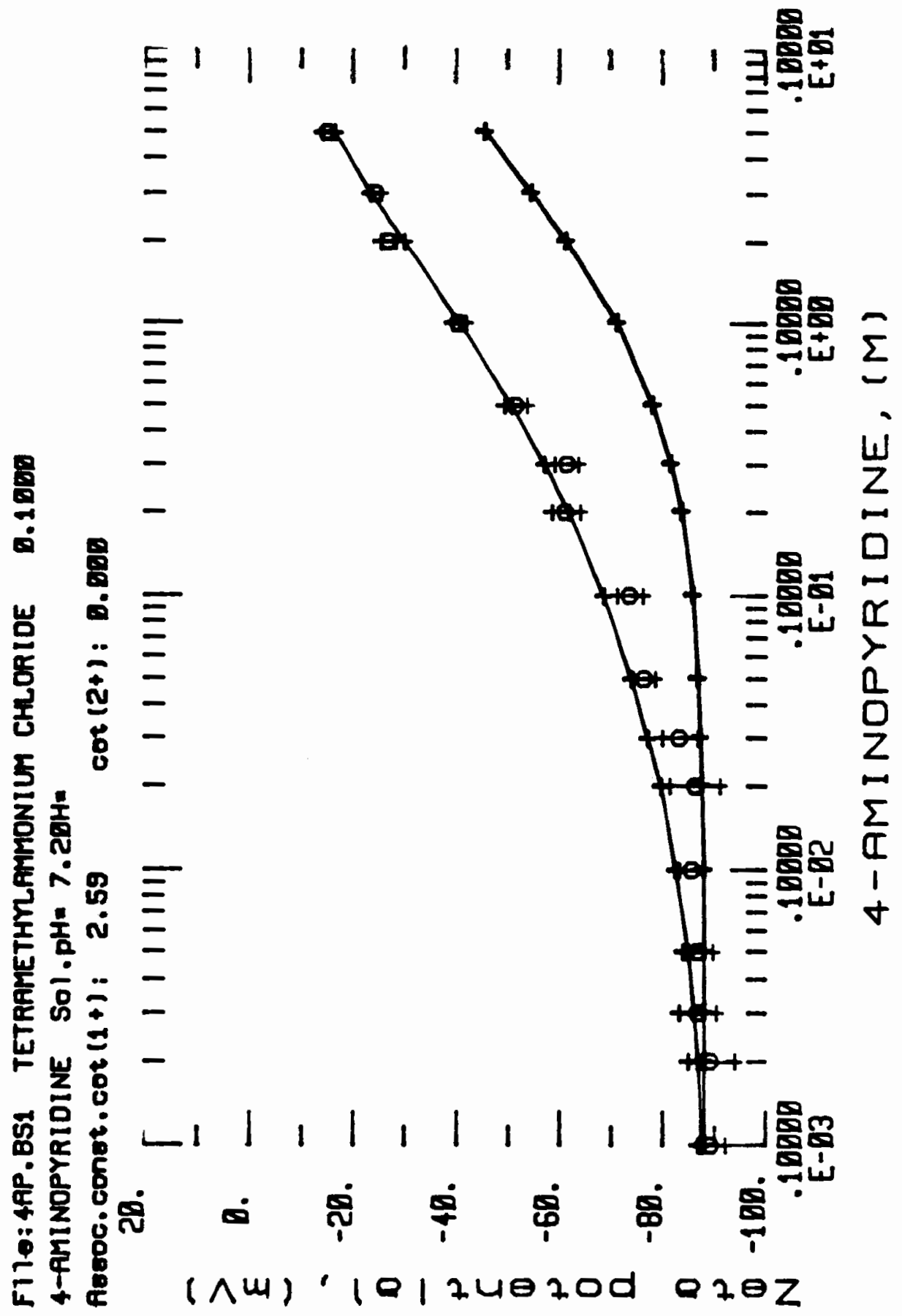

Figure 12. Adsorption isotherm for 4-aminopyridine. 


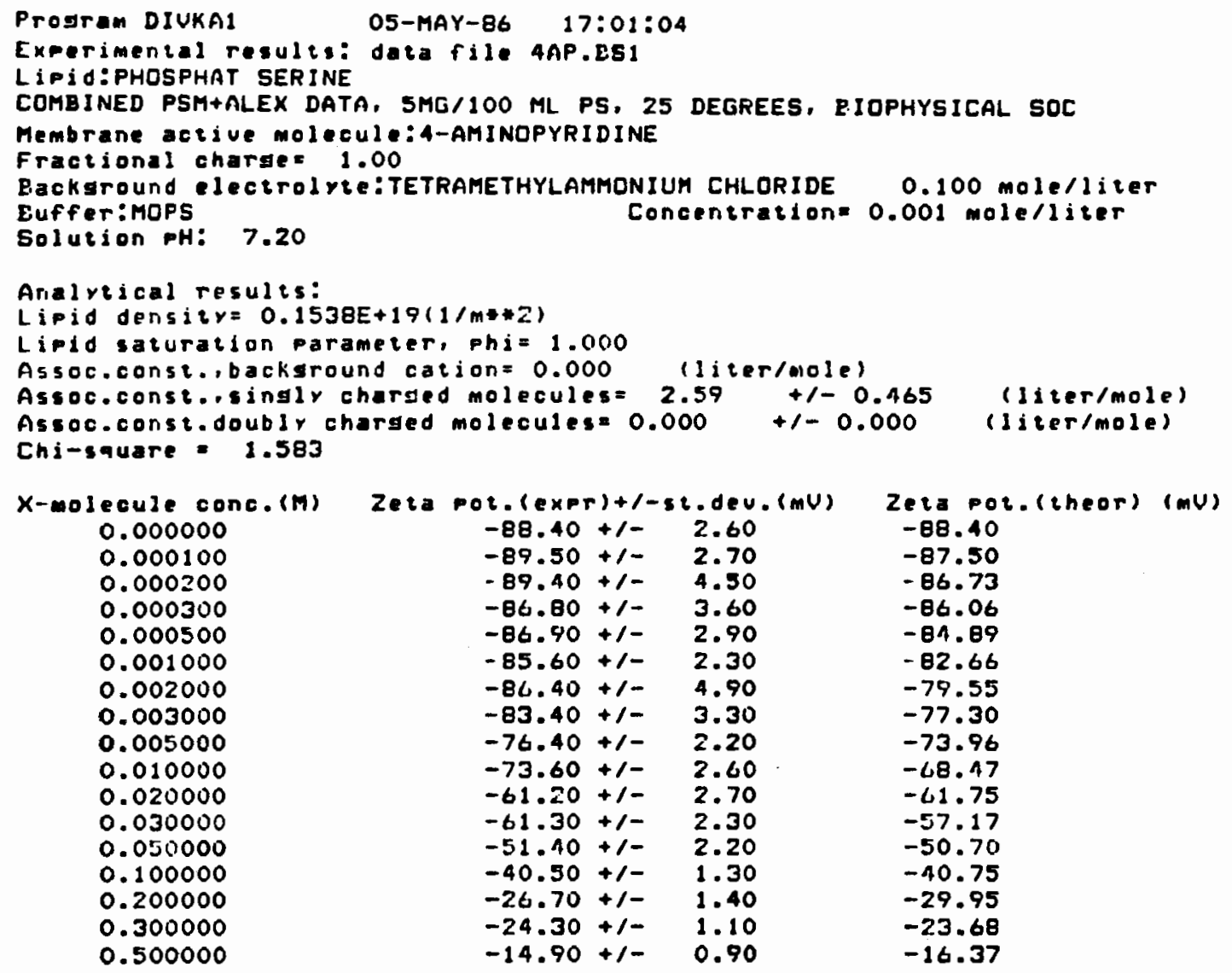




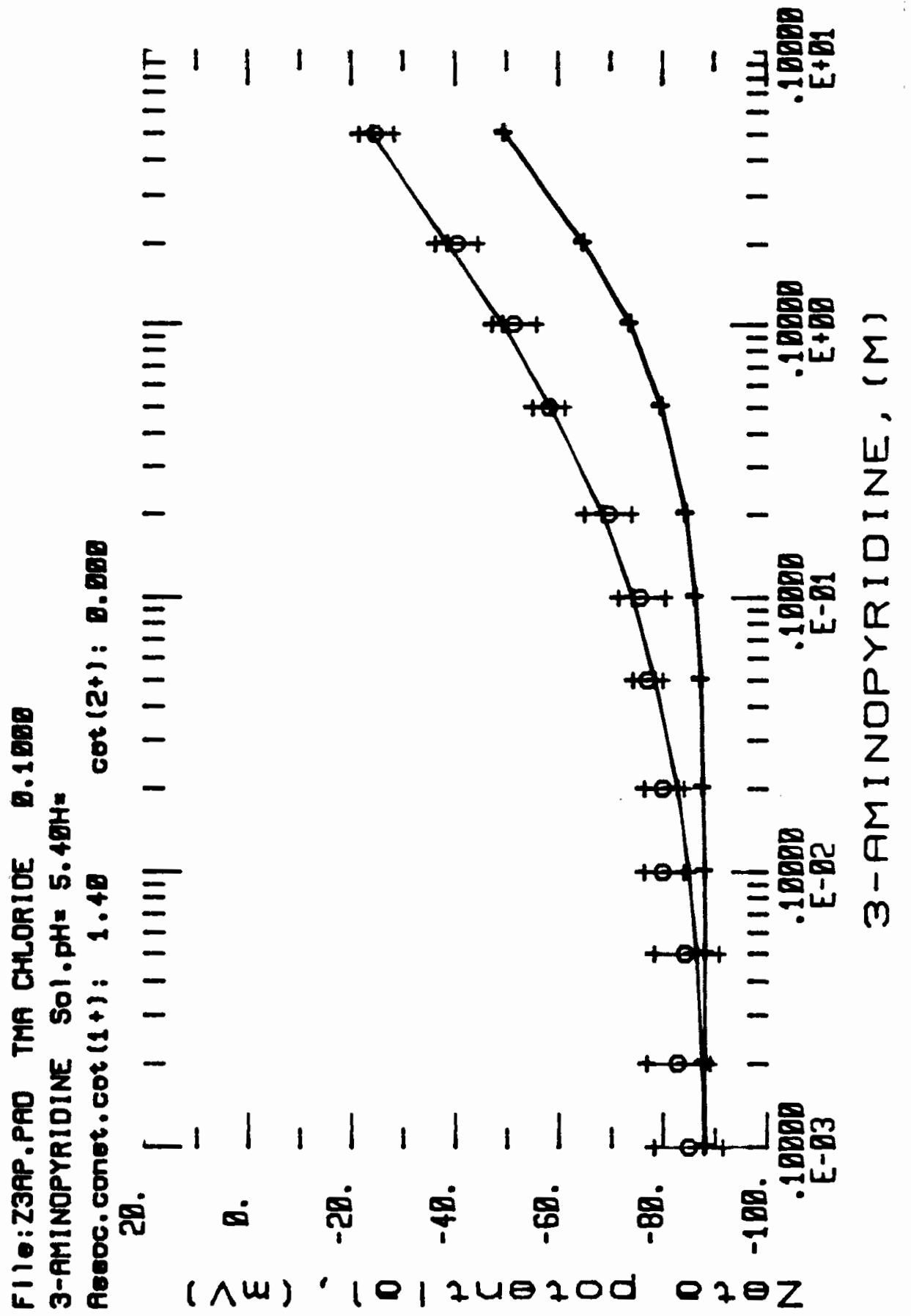

Figure 13. Rdsorption isotherm for 3-aminopyridine (pH 5.4) 


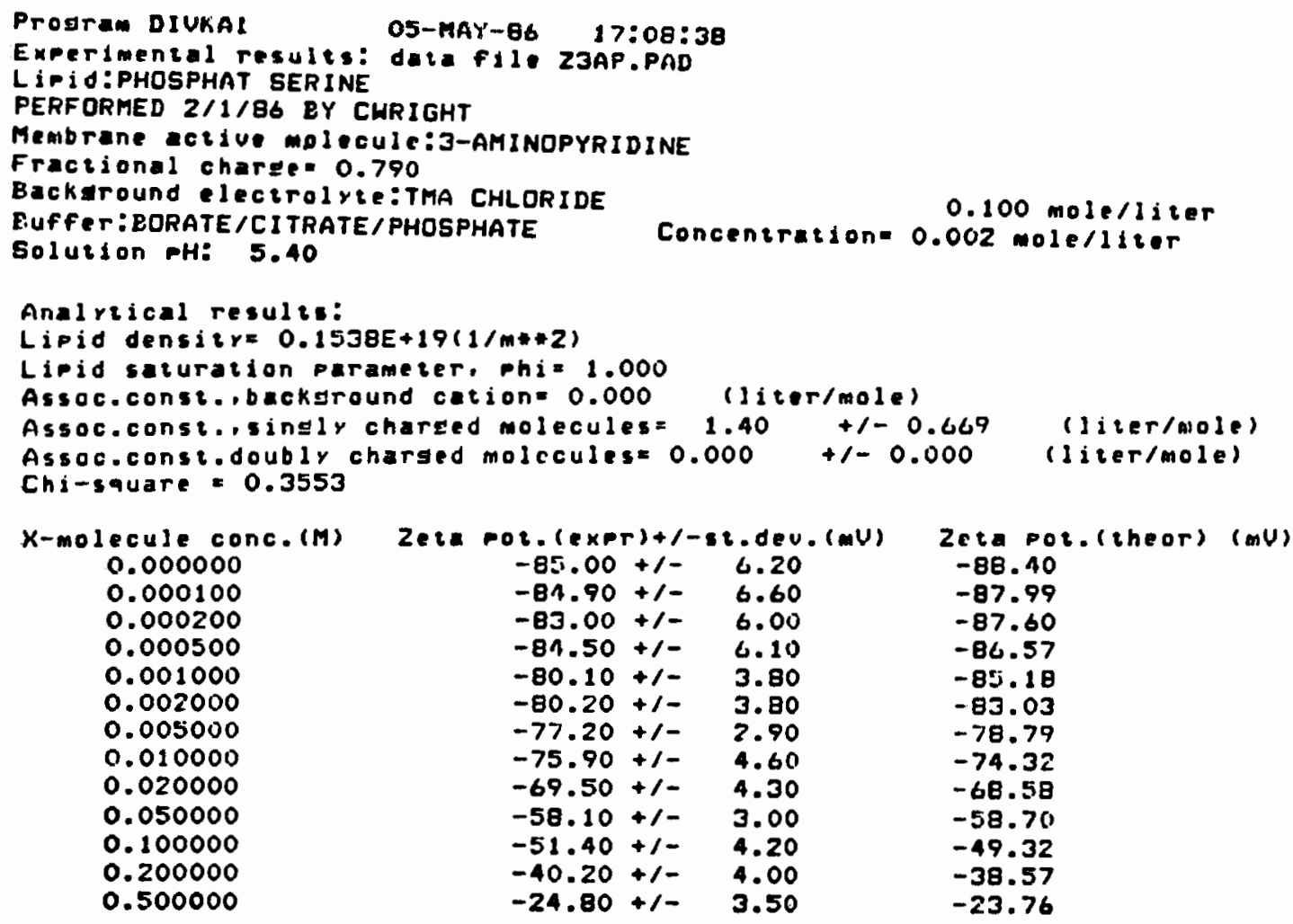




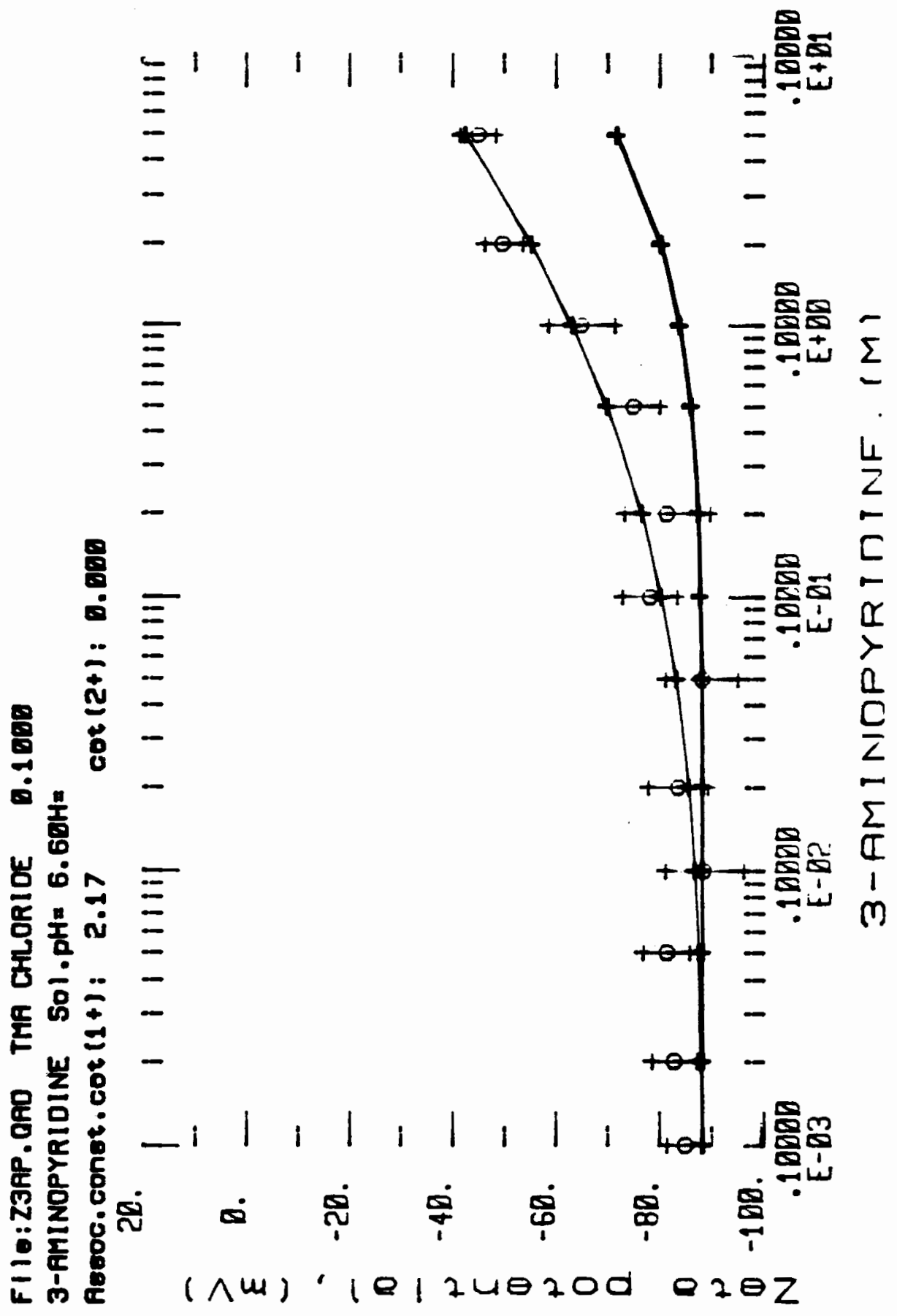

Figure 14. Adsorption isotherm for 3-aminopyridine (PH 6.6). 


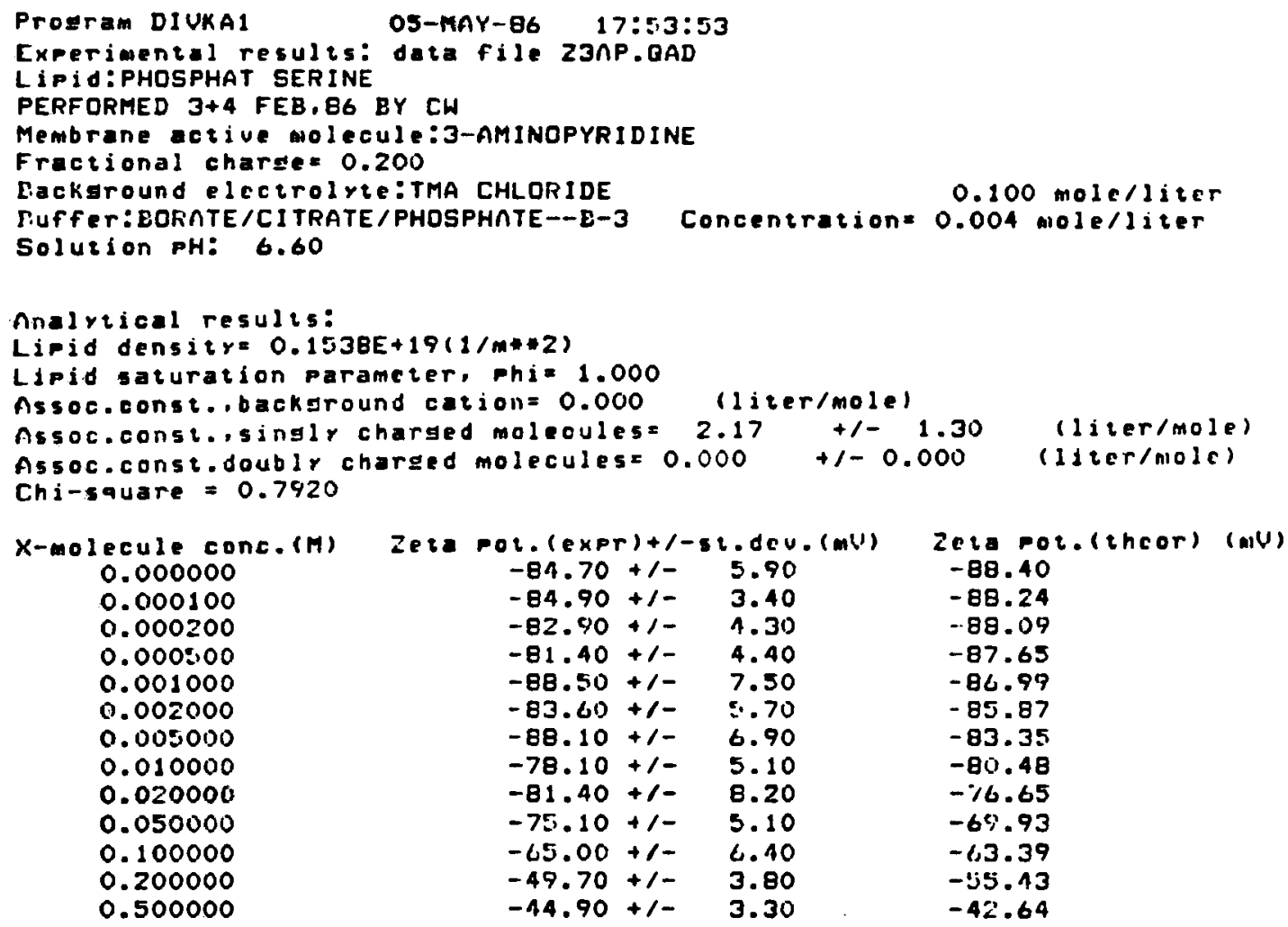




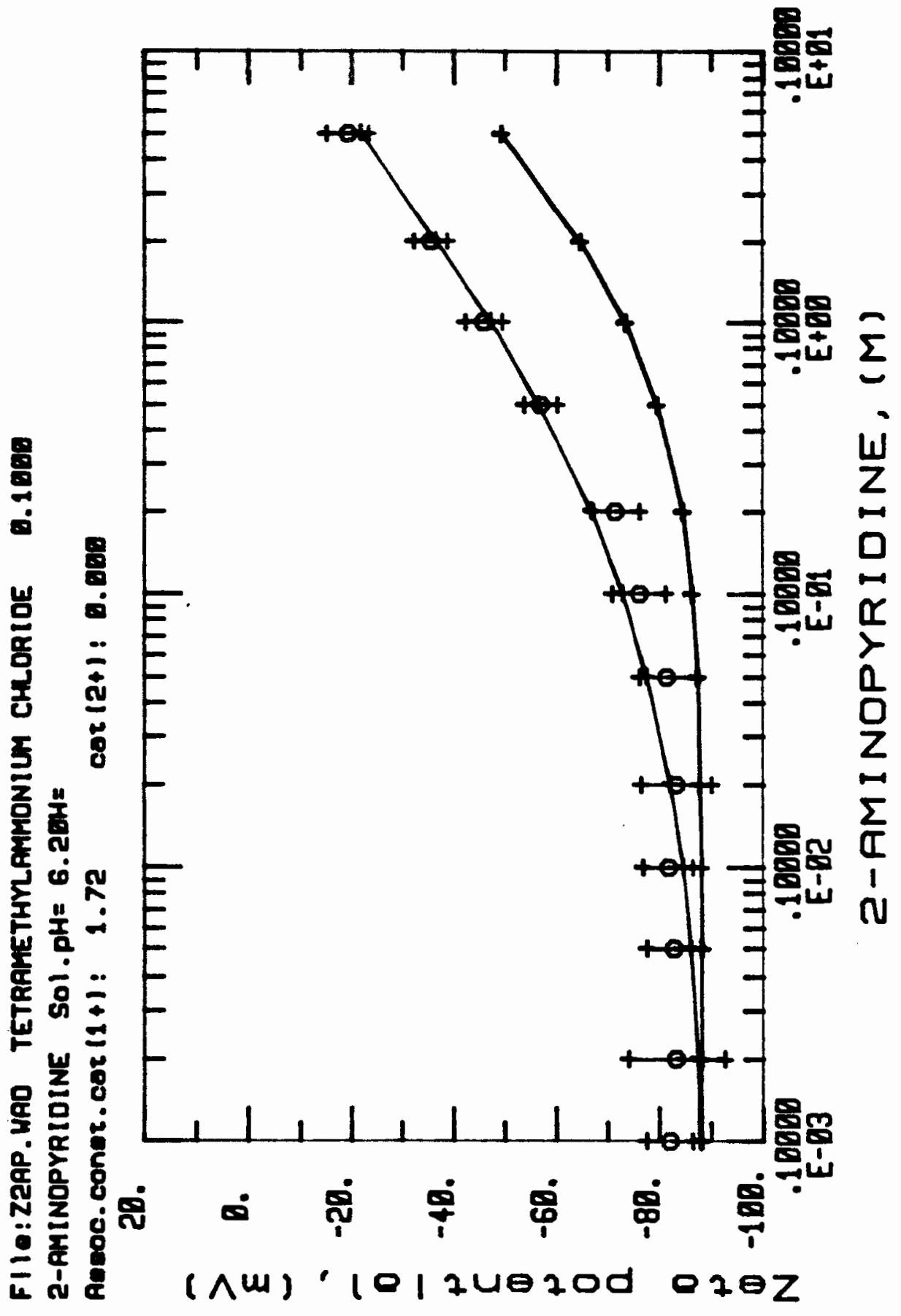

Figure 15. Adsorption isotherm for 2-aminopyridine (pH 6.2). 


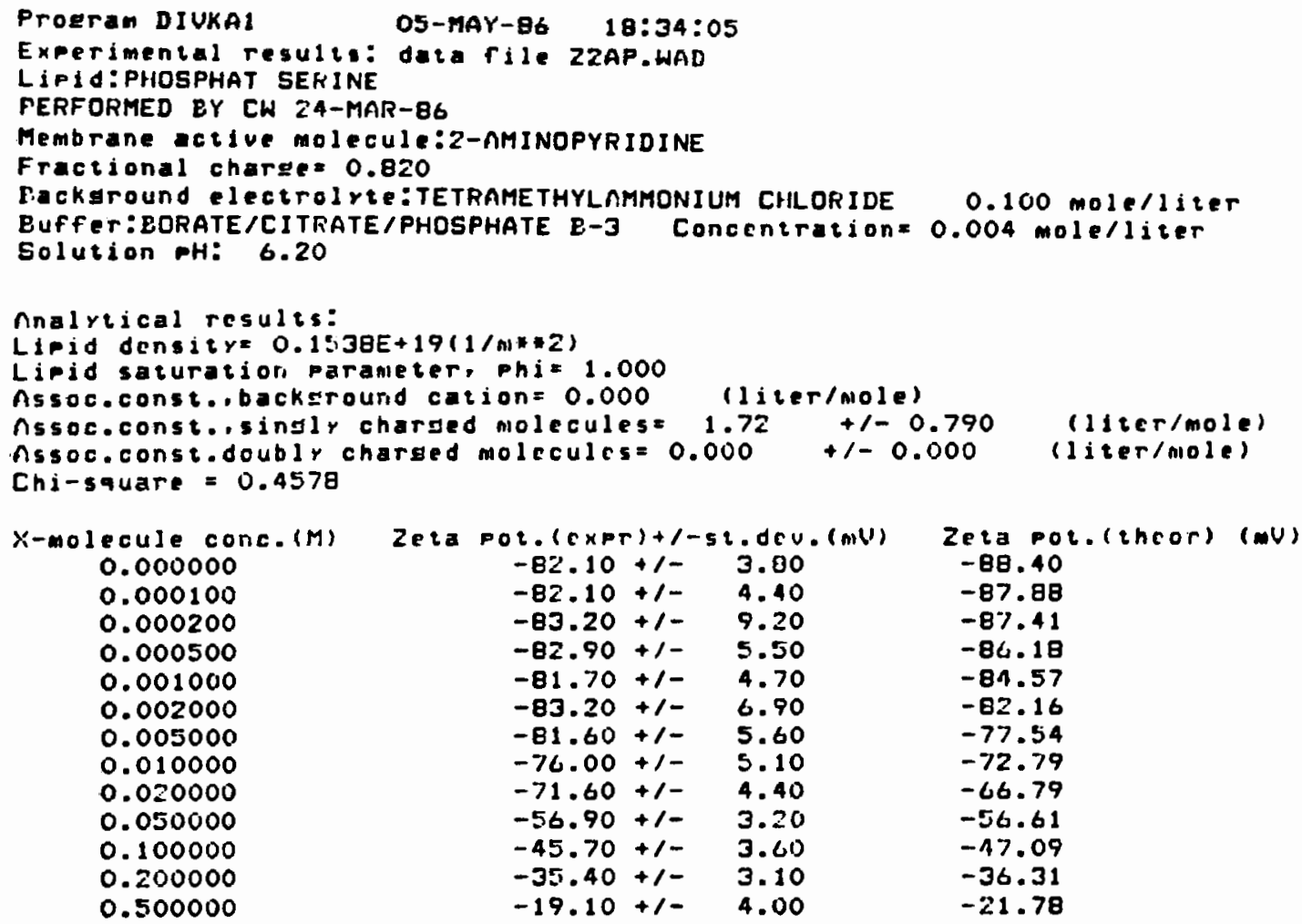




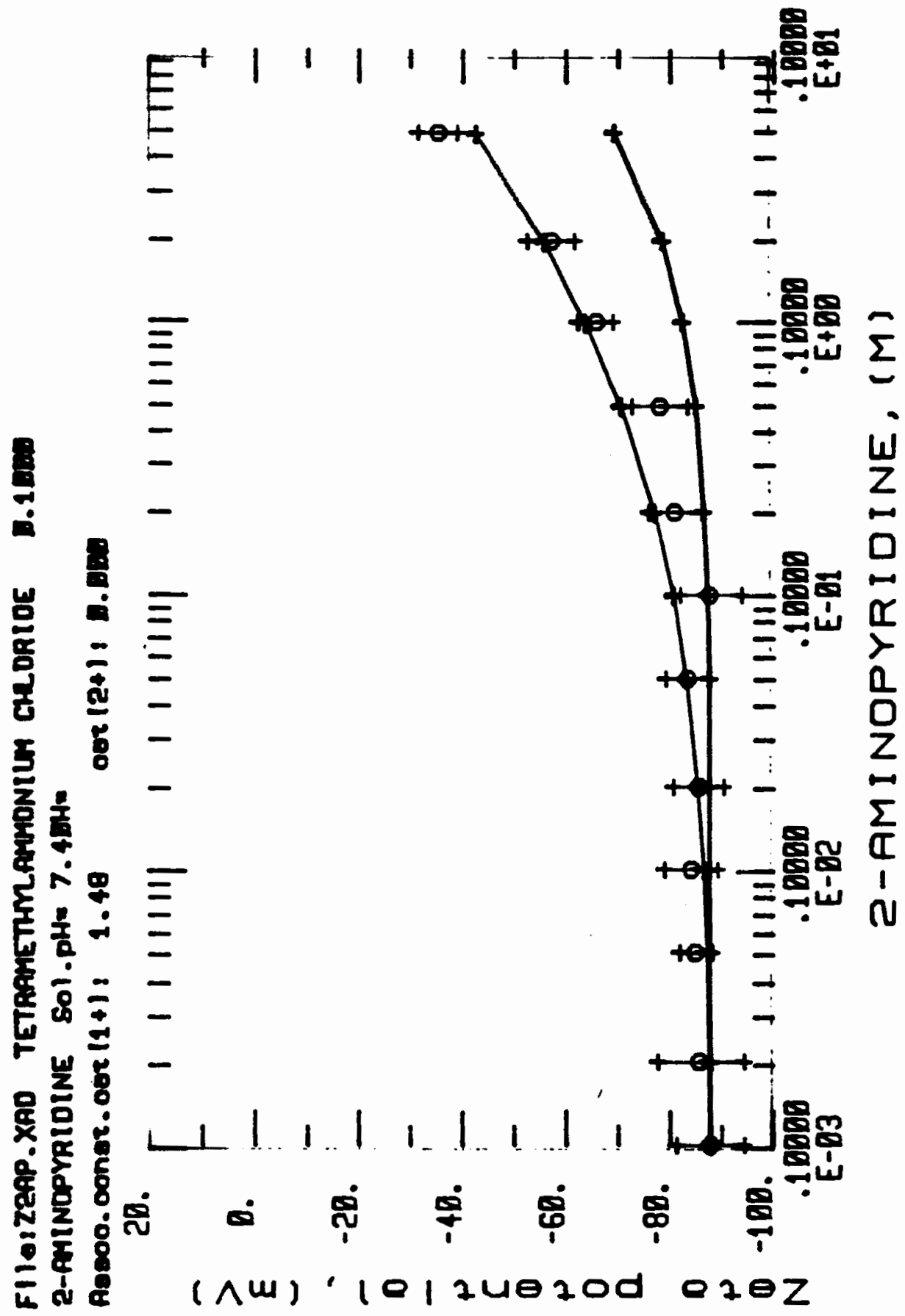
Figure 16. Adsorption isotherm for 2-aminopyridine
(pH 7.4). 


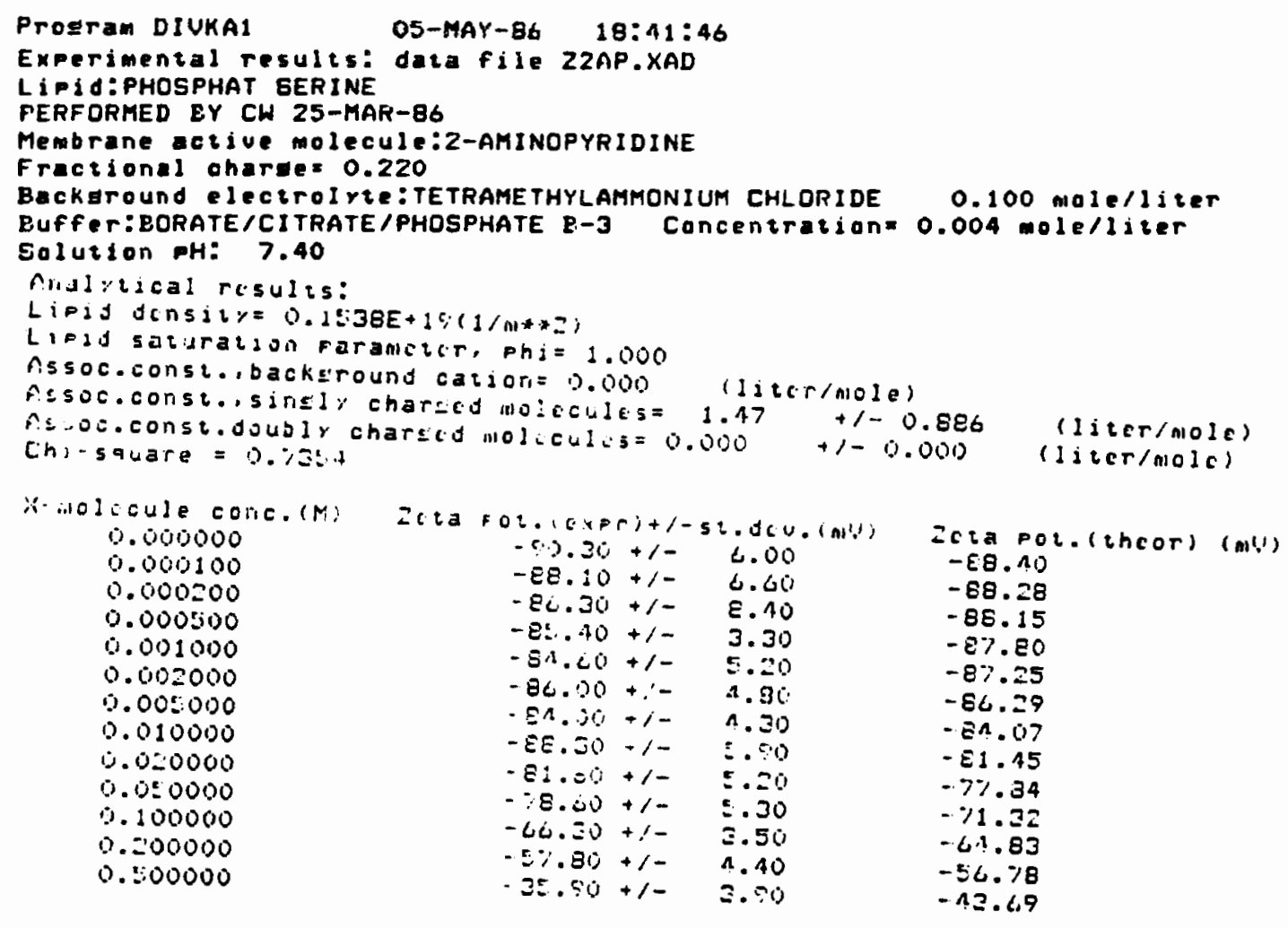




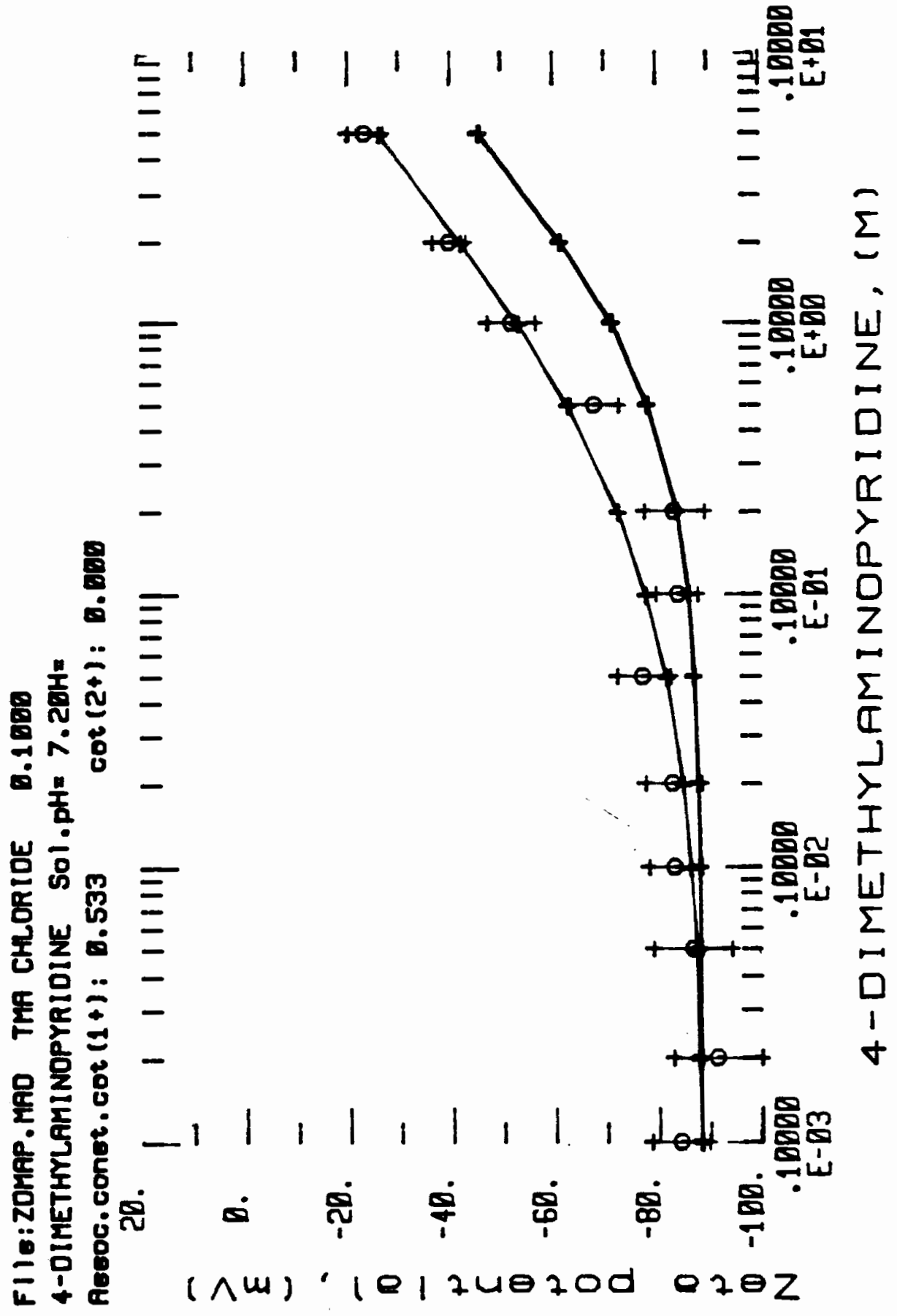

Figure 17. Adsorption isotherm for 4-dimethylaminopridine. 


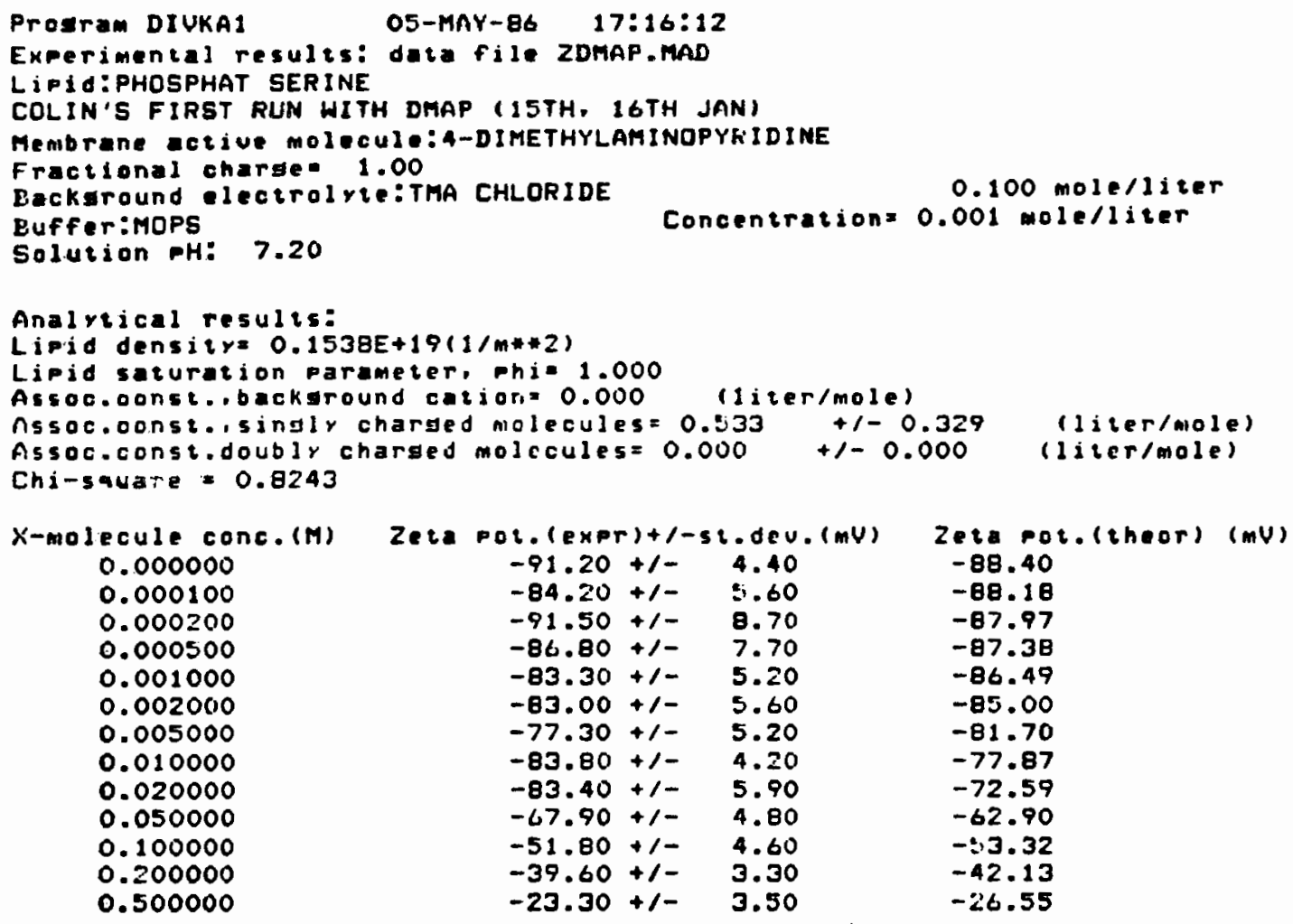




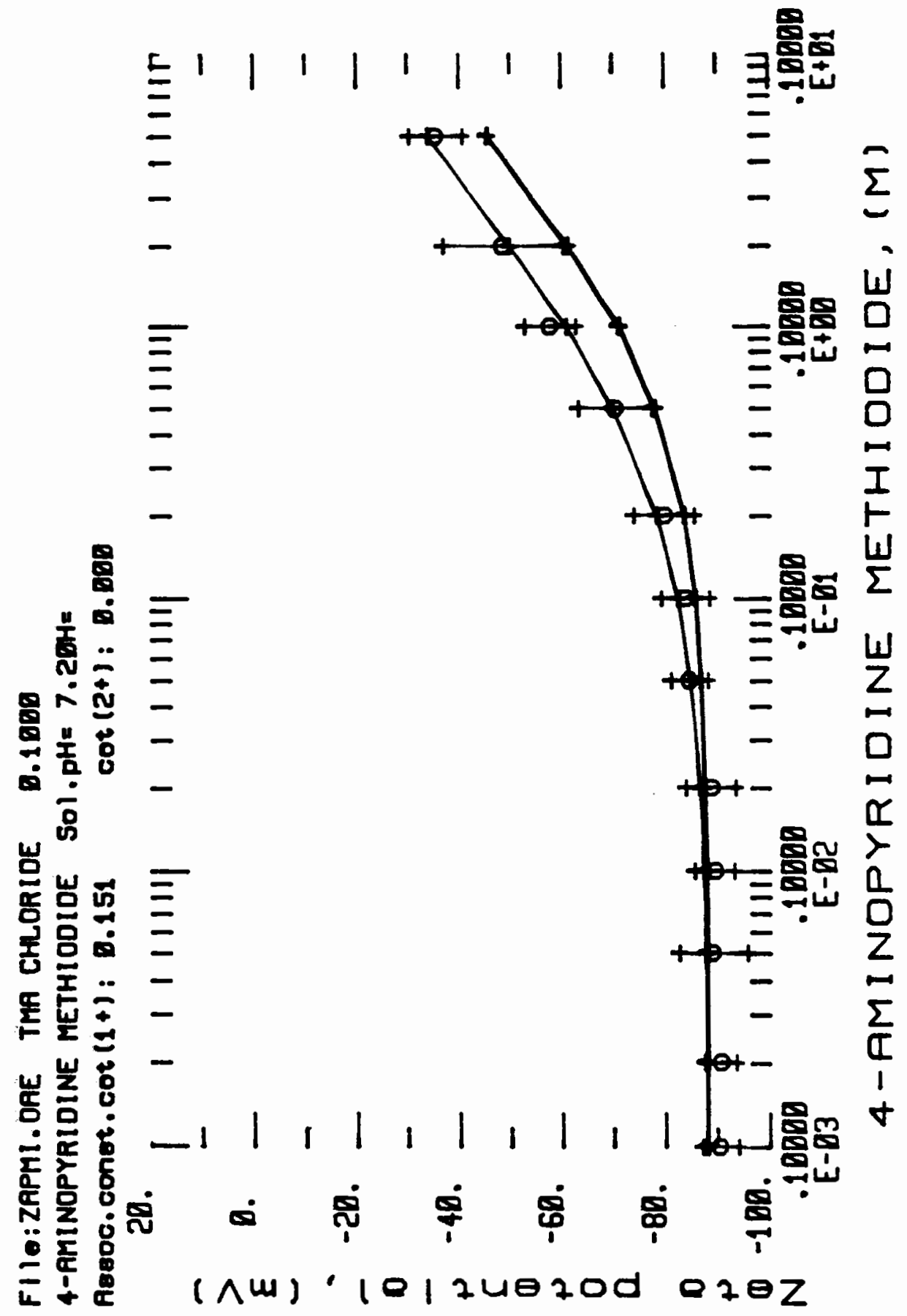
Figure 18. Adsorption isotherm for 4 -aminopyridine
methiodide. 


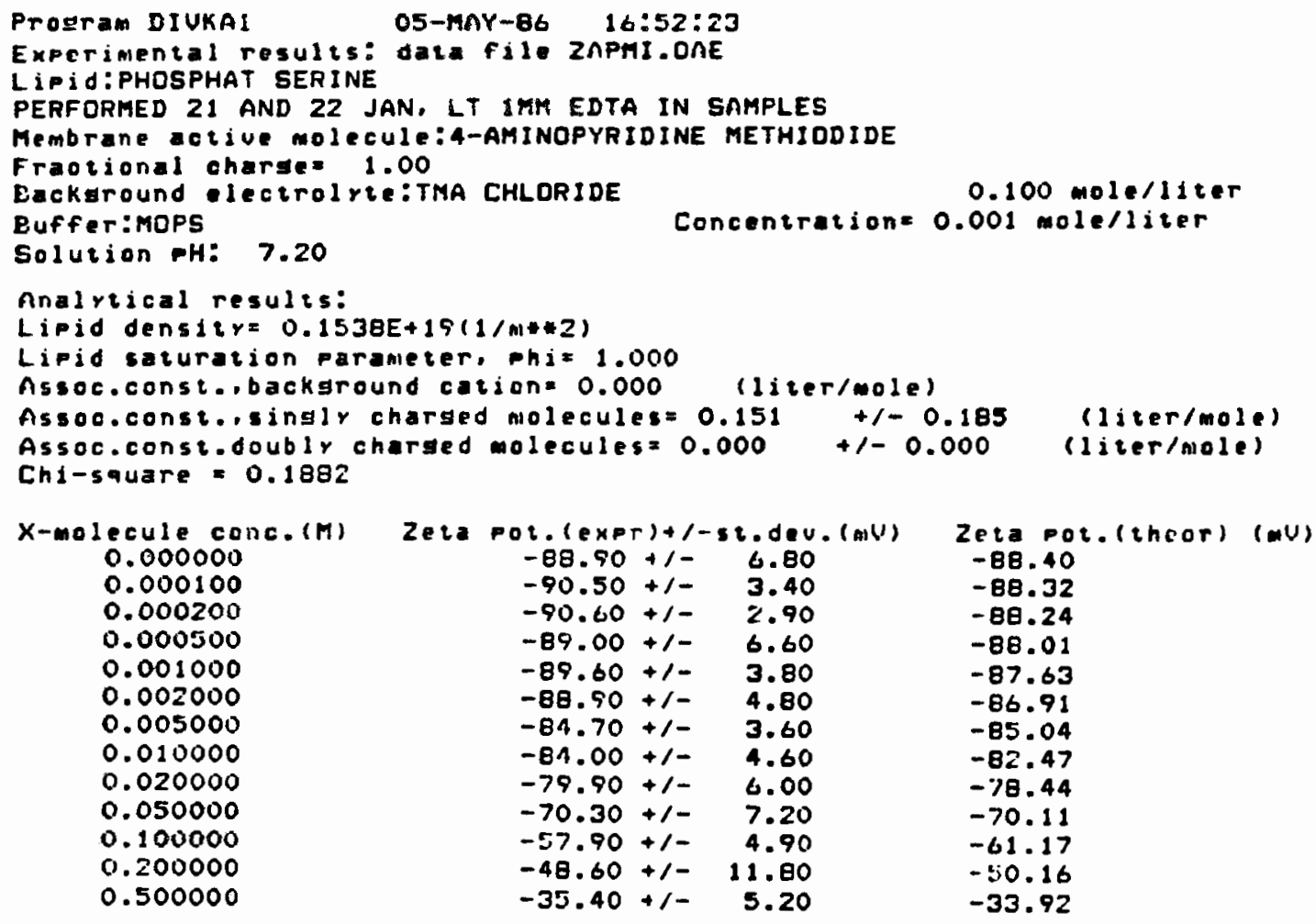

